\title{
NCRs and DNAM-1 mediate NK cell recognition and lysis of human and mouse melanoma cell lines in vitro and in vivo
}

\author{
Tadepally Lakshmikanth, ${ }^{1,2}$ Shannon Burke, ${ }^{3}$ Talib Hassan Ali, ${ }^{1}$ Silvia Kimpfler, ${ }^{4,5}$ Francesco Ursini, ${ }^{1}$ \\ Loredana Ruggeri, ${ }^{6}$ Marusca Capanni, 6 Viktor Umansky,, ${ }^{4,5}$ Annette Paschen, ${ }^{4,5}$ Antje Sucker, ${ }^{4,5}$ \\ Daniela Pende, ${ }^{7}$ Veronika Groh, ${ }^{8}$ Roberto Biassoni, ${ }^{9}$ Petter Höglund, ${ }^{2}$ Masashi Kato, ${ }^{10}$ \\ Kazuko Shibuya, ${ }^{11}$ Dirk Schadendorf, ${ }^{4,5}$ Andrea Anichini, ${ }^{12}$ Soldano Ferrone, ${ }^{13}$ Andrea Velardi, ${ }^{6}$ \\ Klas Kärre, ${ }^{2}$ Akira Shibuya, ${ }^{11}$ Ennio Carbone, ${ }^{1,2}$ and Francesco Colucci ${ }^{3}$
}

\begin{abstract}
1Tumor Immunology Laboratory, Department of Experimental and Clinical Medicine, University of Catanzaro "Magna Graecia", Catanzaro, Italy. 2Department of Microbiology, Tumor and Cell Biology, Karolinska Institutet, Stockholm, Sweden. ${ }^{3}$ Laboratory of Lymphocyte Signalling and Development, The Babraham Institute, Cambridge, United Kingdom. ${ }^{4}$ Skin Cancer Unit, German Cancer Research Center, Heidelberg, Germany. ${ }^{5}$ University Hospital Mannheim, Mannheim, Germany. ${ }^{6}$ Policlinico Monteluce, University of Perugia, Perugia, Italy.

${ }^{7}$ Istituto Nazionale per la Ricerca Sul Cancro, Genoa, Italy. ${ }^{8}$ Fred Hutchinson Cancer Research Center, Seattle, Washington, USA. ${ }^{9}$ stituto Giannina Gaslini, Genoa, Italy. ${ }^{10}$ Department of Biomedical Sciences, Chubu University, Aichi, Japan. ${ }^{11 D e p a r t m e n t ~ o f ~ I m m u n o l o g y, ~ I n s t i t u t e ~ o f ~ B a s i c ~ M e d i c a l ~ S c i e n c e s, ~}$ Graduate School of Comprehensive Human Sciences, and Center for TARA, University of Tsukuba, Tsukuba, Ibaraki, Japan.

${ }^{12}$ Fondazione IRCCS Istituto Nazionale dei Tumori, Milan, Italy. ${ }^{13}$ Department of Surgery, Department of Immunology, and Department of Pathology, University of Pittsburgh Cancer Institute, Pittsburgh, Pennsylvania, USA.
\end{abstract}

\begin{abstract}
NK cells use a variety of receptors to detect abnormal cells, including tumors and their metastases. However, in the case of melanoma, it remains to be determined what specific molecular interactions are involved and whether NK cells control metastatic progression and/or the route of dissemination. Here we show that human melanoma cell lines derived from LN metastases express ligands for natural cytotoxicity receptors (NCRs) and DNAX accessory molecule-1 (DNAM-1), two emerging NK cell receptors key for cancer cell recognition, but not NK group 2 member D (NKG2D). Compared with cell lines derived from metastases taken from other anatomical sites, LN metastases were more susceptible to NK cell lysis and preferentially targeted by adoptively transferred NK cells in a xenogeneic model of cell therapy. In mice, DNAM-1 and NCR ligands were also found on spontaneous melanomas and melanoma cell lines. Interference with DNAM-1 and NCRs by antibody blockade or genetic disruption reduced killing of melanoma cells. Taken together, these results show that DNAM-1 and NCRs are critical for NK cell-mediated innate immunity to melanoma cells and provide a background to design NK cell-based immunotherapeutic strategies against melanoma and possibly other tumors.
\end{abstract}

\section{Introduction}

It is important to decipher the molecular interactions between melanoma cells and various components of the immune system if we are to manipulate the latter for safe and effective therapeutic strategies. Transgenic mice can be used to study spontaneous melanoma (1) and have proven useful in recapitulating aspects of the natural history of the human disease, such as activation of specific proto-oncogenes (2), inactivation of tumor suppressor genes (3), and progression to metastasis (4). Convincing in vivo evidence indicates that $\mathrm{NK}$ cells prevent and control tumor growth and dissemination in mouse models $(5,6)$. The contribution of innate immunity to immunosurveillance of melanoma has recently been studied. Experiments with the murine B16 cell line derived from spontaneous murine melanoma have suggested that NK cells prevent melanoma metastasis in adoptive transfer experiments $(7)$. The revival of the immunosurveillance theory $(8,9)$ has

Authorship note: Tadepally Lakshmikanth, Shannon Burke, Ennio Carbone, and Francesco Colucci contributed equally to this work.

Conflict of interest: The authors have declared that no conflict of interest exists. Nonstandard abbreviations used: B6, C57BL/6; DNAM-1, DNAX accessory molecule-1; E:T, effector/target (ratio); LFA-1, leukocyte function-associated antigen-1; MICA, MHC class I chain-related protein A; NCR, natural cytotoxicity receptor; NKG2D, NK group 2 member D; ULBP1, UL16-binding protein 1.

Citation for this article: J. Clin. Invest. 119:1251-1263 (2009). doi:10.1172/JCI36022. renewed interest in defining the interactions of tumor cells with the host immune system, but there is little information about the role of NK cells in the control of tumor growth in humans. The paucity of this information limits our ability to design rational NK cell-based immunotherapeutic strategies for the treatment of malignant diseases. We have focused on human malignant melanoma, since immunological events are believed to play a role in its pathogenesis and clinical course (10).

NK cells are known to kill virus-infected cells and tumor cells while sparing healthy autologous cells $(11,12)$. The lytic capability of NK cells depends on the integrated balance between activating and inhibitory signals. The latter are generated by the binding of MHC class I molecules to killer cell immunoglobulin-like receptors (KIRs) and to immunoglobulin-like transcript (ILT, also known as LIR, CD85) in humans, to Ly49 in mice, and to the CD94/NKG2A heterodimer $(13,14)$ in both species. NK cells sense signs of infection, stress, and malignant transformation through activating receptors such as NK group 2 member D (NKG2D), which recognizes stress-inducible molecules $\mathrm{MHC}$ class I chain-related protein A (MICA) and MICB and the UL16-binding proteins 1-4 (ULBP1-4, also known as RAET proteins), or through natural cytotoxicity receptors (NCRs) that recognize viral hemagglutinin and as yet undefined tumor cell-associated ligands (14). Other receptors, such as $2 \mathrm{~B} 4$ and DNAX accessory molecule-1 (DNAM-1), bind to consti- 
Table 1

Patient characteristics

$\begin{array}{lcccl}\text { Patient } & \begin{array}{c}\text { Disease } \\ \text { stage }\end{array} & \begin{array}{c}\text { Tumor } \\ \text { site }\end{array} & \text { Cell line } & \text { HLA type } \\ \text { Mel1 } & \text { IV } & \text { LN } & \text { Mel1LN } & \text { A3, B7 (Bw6), B63 (Bw4), Cw7 } \\ & \text { IV } & \text { Pleura } & \text { Mel1PL } & \\ & \text { IV } & \text { Skin } & \text { Mel1SK } & \\ \text { Mel2 } & \text { IV } & \text { Ascite } & \text { Mel2AS } & \text { A1, A24, B8 (Bw6), B27 (Bw4), } \\ & & & & \text { B57 (Bw4), Cw2, Cw6 } \\ \text { Mel3 } & \text { IV } & \text { Pleura } & \text { Mel2PL } & \\ & \text { III } & \text { LN } & \text { Mel3LN1A } & \text { A1, A11, B37 (Bw4), B51 (Bw4) } \\ \text { Mel4 } & \text { IV } & \text { LN } & \text { Mel3LN2A } & \\ & \text { IV } & \text { Skin } & \text { Mel4SK1 } & \text { A2, A3, B8 (Bw6), B62 (Bw6), Cw3, Cw7 } \\ \text { Mel5 } & \text { IV } & \text { Skin } & \text { Mel4SK2 } & \\ & \text { IV } & \text { Skin } & \text { Mel5SK1 } & \text { A1, A2, B8 (Bw6), B44 (Bw4) } \\ \text { Mel6 } & \text { IV } & \text { Skin } & \text { Mel5SK2 } & \\ \text { Mel7 } & \text { IV } & \text { LN } & \text { Mel6LN } & \text { NT } \\ \text { Mel8 } & \text { IV } & \text { Skin } & \text { Mel7LN } & \text { NT } \\ & \text { IV } & \text { Skin } & \text { Mel8SK2 } & \text { A2, A3, B7 (Bw6), B18 (Bw6), Cw1, Cw7 } \\ \text { Mel9 } & \text { III } & \text { LN } & \text { Mel9LN } & \text { A1, B8 (Bw6), B51 (Bw4) } \\ \text { Mel10 } & \text { IV } & \text { Ascite } & \text { Mel10AS } & \text { NT } \\ \text { Mel11 } & \text { IV } & \text { Skin } & \text { Mel11SK } & \text { A3, B7 (Bw6), B39 (Bw6) } \\ \text { Mel12 } & \text { III } & \text { LN } & \text { Mel12LN } & \text { A3, A10, B7 (Bw6), B14 (Bw6) } \\ \text { Mel13 } & \text { IV } & \text { LN } & \text { Mel13LN } & \text { A1, A24 (Bw4), B7 (Bw6), B35 (Bw6) } \\ \text { Mel14 } & \text { III } & \text { Skin } & \text { Mel14SK } & \text { A1, A2, B8 (Bw6), B62 (Bw6) } \\ & \text { IV } & \text { LN } & \text { Mel14LN } & \\ \text { Mel15 } & \text { IV } & \text { LN } & \text { Mel15LN } & \text { NT } \\ \text { Mel16 } & \text { IV } & \text { Skin } & \text { Mel15SK } & \\ & \text { IV } & \text { Skin } & \text { Mel16PR } & \text { A23, A68, B18 (Bw6), B44 (Bw4), } \\ & & & & \text { Cw4, Cw7 } \\ \text { Mel17 } & \text { IV } & \text { LN } & \text { Mel16LN } & \\ \text { Mel18 } & \text { IV } & \text { Skin } & \text { Mel16SK } & \\ & \text { III } & \text { LN } & \text { Mel17LN } & \text { NT } \\ & & & \text { Mel18LN } & \text { NT } \\ \text { Cell } & & \\ & & & & \\ & & & \end{array}$

Cell line abbreviations are a combination of the patient number and location of the tumor. -AS, ascite melanoma cell line; -PL, pleura metastatic cell line; -SK, skin metastatic cell line; PR, primary skin melanoma. NT, not tested. ${ }^{A}$ Mel3LN1 and Mel3LN2 indicate metastatic cell lines taken from different parts of the same anatomical site. BPrimary skin melanoma.

Here we investigate whether human metastatic melanoma cells isolated from different anatomical sites are susceptible to NK cell recognition. The results suggest that $\mathrm{LN}$ metastases are preferentially targeted by both autologous and allogeneic NK cells in vitro, compared with metastases from other sites (including skin, pleura, and ascites) or from hematogenous metastases. Human melanoma metastases and mouse primary tumors, as well as mouse melanoma cell lines, shared the expression of DNAM-1 and NCR ligands and low MHC class I expression. Disruption of DNAM-1 and NCR interactions with their ligands by blockade or by genetic means in knockout mice reduced NK cell recognition in vitro and in vivo in both species. These results have implications for the design of immunotherapeutic strategies based on targeting metastases in the "sentinel" LN in patients with melanoma and for reliance on the administration of allogeneic NK cells or on the potentiation of autologous NK cells.

\section{Results}

Human melanoma cell lines express ligands for NCRs and DNAM-1. We used soluble recombinant NKp30-Fc, NKp44-Fc, and NKp46-Fc to measure the expression of NCR ligands on 6 cell lines derived from LN metastases and 6 cell lines derived from skin metastases of 7 melanoma patients. The patients' characteristics are summarized in Table 1. While the NKp30-Fc staining was low and inconsistent across all samples, NKp44-Fc and NKp46-Fc stained 6 of 6 LN metastases. In 2 of 7 patients (Mel1 and Mel16) we were able to analyze paired LN and skin metastases from the same patient. This direct comparison clearly showed brighter NKp44-Fc

tutively expressed ligands CD48 and poliovirus receptor (CD155) or nectin-2 (CD112), respectively (15). Cytotoxicity and cytokine production are triggered in resting NK cells by the simultaneous engagement of receptor pairs such as NKp46 and DNAM-1 (16). DNAM-1 is emerging as a key co-activating receptor in immunity to human cancer. In neuroblastoma (17), ovarian carcinoma (18), and hematopoietic malignancies (19), DNAM-1 participates in cancer cell recognition together with NCRs and, to a lesser extent, NKG2D. Mouse DNAM-1 is a crucial component of T cell-mediated immunological surveillance and partially contributes to NK cell-mediated lymphoma rejection (20), but its relevance for NK cell immunity to melanoma in vivo is unknown. NCRs are key receptors in recognition of human cancer cells, although the tumor antigens they recognize are still unknown. The absence of one such receptor (NKp46) did impair the rejection of lymphoma cells in mice, although the defect was strain dependent (21). Very recently, NKp46 ligands have been shown on benign and malignant human melanocytes (22), although the functional relevance of this finding remains to be determined. Therefore, the molecular mechanisms underlying NK cell recognition of melanoma cells are largely unknown. and NKp46-Fc staining of LN metastases in both cases (Figure 1A). The NKp44-Fc and NKp46-Fc fluorescence intensity of the staining on LN metastases was significantly brighter than that on skin metastases $(P<0.0005$ and $P<0.05$, respectively) (Figure $1 \mathrm{~B}$; data on the other 5 patients can be found in Supplemental Figure 1A; supplemental material available online with this article; doi:10.1172/JCI36022DS1), suggesting that the ligands for NKp44 and NKp46 are generally expressed on melanoma cells in LN metastases, but less so, or not at all, in skin metastases.

To study the expression of ligands for DNAM-1, NKG2D, and leukocyte function-associated antigen-1 (LFA-1), we used monoclonal antibodies specific for poliovirus receptor (CD155), nectin-2 (CD112), MICA, ULBP1-4, and ICAM-1 on 5 cell lines derived from LN metastases and 6 cell lines derived from metastases of various anatomical sites (including skin, pleura, ascites, and peritoneum) of 5 patients. CD155 and CD112 were expressed on all samples, although the expression of CD112 was low on 5 of 11 samples. By contrast, most NKG2D ligands were either undetectable or were expressed at low levels. Thus, ULBP1, -2 , and -4 were not detected in any of the 11 samples, and ULBP3 and MICA displayed low 
A

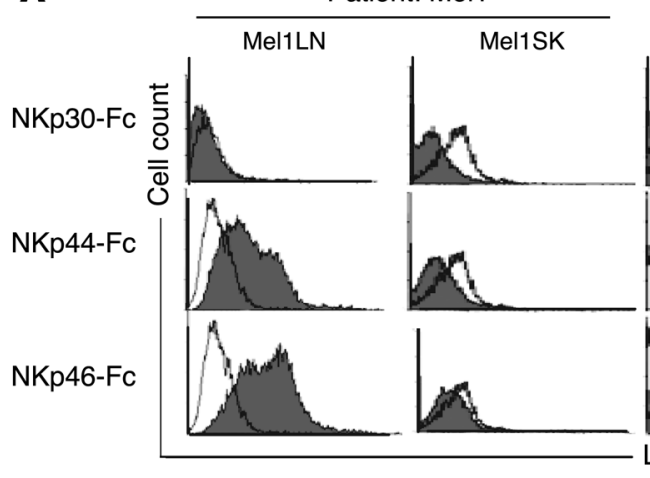

Patient: Mel16

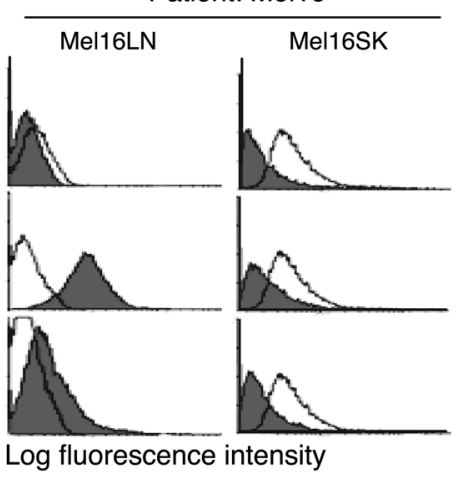

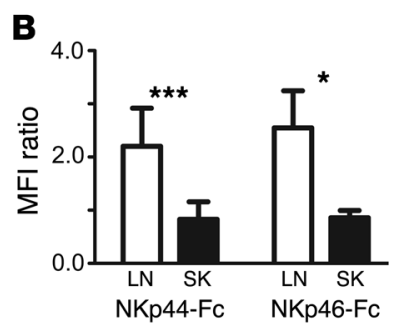

C Patient: Mel1

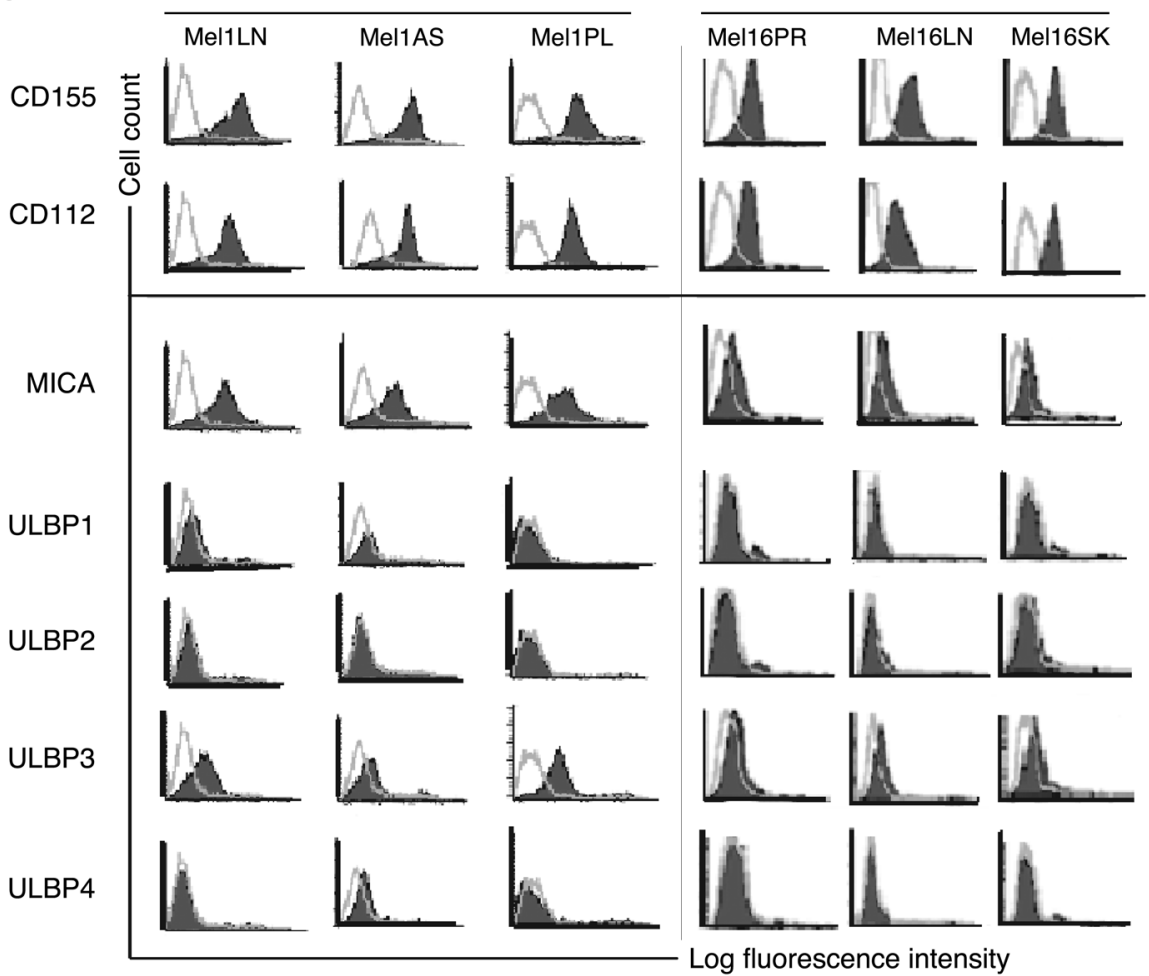

Figure 1

Expression of ligands for NCRs and DNAM-1 on human melanoma metastatic cells. (A) NCR ligand expression was measured by flow cytometry on paired LN and skin (SK) melanoma cell lines obtained from metastases of patients Mel1 and Mel16. Staining with NKp30-Fc, NKp44-Fc, and NKp46-Fc (filled histograms) was compared with staining with irrelevant CD5-Fc (open histograms). (B) Quantification and statistical analysis of NCR ligand expression on 6 unpaired LN (white bars) and skin (black bars) metastatic cell lines from 7 patients measured as fluorescence ratio calculated as MFI (mean + SD) of NKp44-Fc and NKp46-Fc staining over MFI of negative control (CD5-Fc staining). ${ }^{*} P<0.05,{ }^{* * \star} P<0.0005$. (C) DNAM-1 and NKG2D ligand expression (filled histograms) measured by flow cytometry on melanoma cells obtained from different anatomical sites of patients Mel1 and Mel16. Negative controls were stained with FITC-conjugated anti-mouse Ig xenoantibodies (open histograms). Histograms are representative of 2-5 independent experiments. AS, ascite metastasis; PL, pleural metastasis; PR, primary skin melanoma.

expression on 4 and on 5 of the 11 samples, respectively (Figure 1C and Supplemental Figure 1B). The low or absent expression of the NKG2D ligands does not reflect their shedding from the cell surface, since soluble MICA was not detected in any of the supernatants harvested from 14 melanoma cell lines (data not shown). The LFA-1 ligand ICAM-1 was expressed on all melanoma metastatic cell lines regardless of their anatomical origin. HLA class I molecules were expressed at a lower levels than on healthy melanocytes and autolo- gous lymphocytes, regardless of their anatomical origin (Supplemental Figure 2). Collectively, these data suggest that, regardless of the anatomic site of origin, melanoma metastases express low levels of HLA class I molecules. Furthermore, they express ligands for DNAM-1 and LFA-1 but do not generally express ligands for NKG2D. Lastly, LN metastases preferentially express ligands for NKp44 and NKp46. A summary of NKG2D, DNAM-1, and LFA-1 ligand expression on melanoma cell lines is shown in Table 2. 
Table 2

Flow cytometry analysis of NKG2D and DNAM-1 ligands and ICAM-1 expression in human melanoma cell lines

\begin{tabular}{|c|c|c|c|c|c|c|c|c|c|c|c|}
\hline \multirow[t]{2}{*}{ Patient } & \multirow{2}{*}{$\begin{array}{l}\text { Cell } \\
\text { line }\end{array}$} & \multirow{2}{*}{$\begin{array}{l}\text { Tumor } \\
\text { type }\end{array}$} & \multicolumn{2}{|c|}{ DNAM-1 ligands } & \multicolumn{6}{|c|}{ NKG2D ligands } & \multirow[t]{2}{*}{ ICAM-1 } \\
\hline & & & PVR & Nectin-2 & MICA & MICA/B & ULBP1 & ULBP2 & ULBP3 & ULBP4 & \\
\hline \multirow[t]{3}{*}{ Mel1 } & Mel1LN & LN & $97 \pm 27$ & $47 \pm 12$ & $47 \pm 7$ & $36 \pm 4$ & $0.7 \pm 0.3$ & $4.7 \pm 4.0$ & $5.7 \pm 2.0$ & $0.25 \pm 0.25$ & $314 \pm 129$ \\
\hline & Mel1PL & Pleura & $77 \pm 23$ & $28 \pm 7$ & $25 \pm 4$ & $30 \pm 1.4$ & $0.6 \pm 0.4$ & $0.2 \pm 0.2$ & $3.8 \pm 1.0$ & $0.75 \pm 0.75$ & $156 \pm 72$ \\
\hline & Mel1SK & Skin & $88 \pm 32$ & $44 \pm 15$ & $38 \pm 6$ & $41 \pm 2$ & 0 & $0.3 \pm 0.3$ & $13 \pm 3$ & $0.5 \pm 0.5$ & $385 \pm 158$ \\
\hline \multirow[t]{2}{*}{ Mel2 } & Mel2AS & Ascite & $89 \pm 56$ & $55 \pm 31$ & $2 \pm 0.4$ & $1.5 \pm 0.5$ & $3.2 \pm 0.2$ & $1 \pm 1$ & $13 \pm 3$ & $0.5 \pm 0.5$ & $31 \pm 24$ \\
\hline & Mel2PL & Pleura & $123 \pm 69$ & $71 \pm 37$ & $2 \pm 0.7$ & $5 \pm 2$ & $1 \pm 0$ & $2 \pm 1$ & $22 \pm 6$ & $0.5 \pm 0.5$ & $56 \pm 32$ \\
\hline \multirow[t]{2}{*}{ Mel3 } & Mel3LN1 & LN & $41 \pm 8$ & $6 \pm 1$ & $9 \pm 3$ & $12 \pm 3$ & $1 \pm 0.4$ & $0.5 \pm 0.5$ & $1 \pm 0.7$ & $2 \pm 1$ & $29 \pm 11$ \\
\hline & Mel3LN2 & LN & $27 \pm 12$ & $5 \pm 3$ & $6 \pm 1$ & $8 \pm 3$ & $0.2 \pm 0.2$ & $0.2 \pm 0.2$ & $0.2 \pm 0.2$ & $0.5 \pm 0.5$ & ND \\
\hline \multirow[t]{2}{*}{ Mel4 } & Mel4SK1 & Skin & $19 \pm 3$ & $8 \pm 2$ & 0 & 0 & 0 & 0 & 0 & 0 & 0 \\
\hline & Mel4SK2 & Skin & $34 \pm 2$ & $11 \pm 0$ & 0 & 0 & 0 & 0 & 0 & 0 & 0 \\
\hline \multirow[t]{2}{*}{ Mel5 } & Mel5SK1 & Skin & $23 \pm 4$ & $11 \pm 2$ & ND & ND & ND & ND & ND & ND & $48 \pm 24$ \\
\hline & Mel5SK2 & Skin & $25.5 \pm 1.8$ & $6.8 \pm 2.4$ & $9 \pm 3.8$ & ND & $7.3 \pm 2.0$ & $6 \pm 2.2$ & $6 \pm 1.9$ & $5.8 \pm 2.2$ & $34.5 \pm 4.0$ \\
\hline Mel6 & Mel6LN & LN & $33 \pm 6$ & $19 \pm 3$ & $7 \pm 2$ & $7 \pm 3$ & 0 & 0 & 0 & 0 & $6 \pm 4$ \\
\hline Mel7 & Mel7LN & LN & $17 \pm 5$ & $15 \pm 1.5$ & ND & ND & ND & ND & ND & ND & $2 \pm 3$ \\
\hline \multirow[t]{2}{*}{ Mel8 } & Mel8SK1 & Skin & $15 \pm 3$ & $3 \pm 0.8$ & $2 \pm 0.6$ & $4 \pm 2$ & 0 & 0 & 0 & 0 & $19 \pm 5$ \\
\hline & Mel8SK2 & Skin & $28 \pm 5$ & $5 \pm 1$ & $6 \pm 0.4$ & $6 \pm 0.7$ & 0 & 0 & 0 & 0 & $8 \pm 4$ \\
\hline \multirow[t]{2}{*}{ Mel15 } & Mel15LN & LN & $23 \pm 2.1$ & $3.7 \pm 0.0$ & $4.7 \pm 0.4$ & ND & $3.4 \pm 0.4$ & $3.6 \pm 0.5$ & $3.4 \pm 0.2$ & $3.2 \pm 0.2$ & $16.9 \pm 0.0$ \\
\hline & Mel15SK & Skin & $19 \pm 0.3$ & $4.5 \pm 0.1$ & $4.6 \pm 0.4$ & ND & $3.2 \pm 0.7$ & $3.3 \pm 0.6$ & $3.6 \pm 1.1$ & $3.3 \pm 0.7$ & $15.1 \pm 1.9$ \\
\hline \multirow[t]{4}{*}{ Mel16 } & Mel16PR & Skin ${ }^{A}$ & $19 \pm 3$ & $12 \pm 2$ & $2 \pm 1$ & $1.6 \pm 1.0$ & 0 & 0 & $1.2 \pm 0.9$ & 0 & $5 \pm 0.8$ \\
\hline & Mel16LN & LN & $19 \pm 3.2$ & $10 \pm 2$ & $3 \pm 1$ & $1.5 \pm 0.9$ & 0 & 0 & $0.6 \pm 0.6$ & 0 & $21 \pm 7$ \\
\hline & Mel16SK & Skin & $19 \pm 4$ & $12 \pm 3.7$ & $2 \pm 1$ & $1.3 \pm 0.8$ & 0 & 0 & $1 \pm 1$ & 0 & $4 \pm 2$ \\
\hline & Melanocytes ${ }^{B}$ & & 27.7 & 26.9 & 30.1 & ND & 0 & 0 & 6 & 0 & ND \\
\hline
\end{tabular}

ND, not determined. Data reported for patients represent mean \pm SEM of MFI values obtained from 5 independent experiments performed on each cell line. APrimary skin melanoma. BData reported for melanocytes represent mean MFI obtained from 1 representative experiment.

We then analyzed the expression of NK cell ligands in melanoma metastases by immunohistochemical staining of biopsies. In line with the flow cytometry data obtained using metastatic cell lines, we readily detected DNAM-1 ligands and the staining, with CD155 colocalizing with markers of melanoma cells (Supplemental Figure 3). By contrast, we were not able to detect staining of biopsies of LN or skin metastases with NKp44-Fc and with NKp46-Fc (data not shown). However, these negative results do not exclude the expression, although at a low level, of NCR ligands in metastases, given the low level of staining of melanoma cell lines obtained with the NKp44-Fc and NKp46-Fc reagents (Figure 1A). Some of the samples stained positive for MICA/B, and melanocytes also were positive for MICA (Table 2). Among the infiltrating leukocytes, we detected rare $\mathrm{CD}^{-} \mathrm{CD} 56^{+} \mathrm{NK}$ cells, the majority of infiltrating lymphocytes being $\mathrm{CD}^{+}{ }^{+} \mathrm{CD} 56^{-} \mathrm{T}$ cells (Supplemental Figure 3 ).

Primary mouse melanoma tumors and cell lines express ligands for NKp46 and DNAM-1. We then turned to a mouse model of spontaneous melanoma (metallothionein/ret transgenic mice, hereafter referred to as RET mice) to assess whether NCR and DNAM-1 ligands are indeed expressed in primary melanoma tumors. The mouse genome has only 1 NCR gene, Norl, which encodes the NKp46 receptor. As for human NCRs, the tumor-associated cellular ligands are unknown, thus we used an $\mathrm{Fc}$ fusion protein to detect NKp46 ligand expression. Cell suspensions were prepared from tumor lesions of 4 individual RET mice that displayed similar stages of disease progression, as judged by tumor size. These cell suspensions contained $\mathrm{CD} 45^{+}$infiltrating leukocytes, among them rare $\mathrm{CD}^{-} \mathrm{NK} 1.1^{+} \mathrm{NK}$ cells $(0.4 \% \pm 0.3 \%, n=3)$, mirroring the scenario in human biopsies of metastatic lesions. Melanoma cells were included in the CD45- cells. NKp46-Fc stained CD45- cells in 4 of 4 tumors (Figure 2A), whereas NKG2D-Fc stained none.
CD112 was almost undetectable, whereas CD155 was expressed by 4 of 4 tumors (Figure 2A). MHC class I antigens were detected on only 1 of the 4 tumors, and at low levels. Infiltrating leukocytes did not stain for NKp46-Fc and NKG2D-Fc ligands, but expressed DNAM-1 ligands and MHC class I antigens (Figure 2A).

Mouse melanoma cell lines have been used for a long time in clinically relevant models of transplantable melanoma. However, the ligands expressed by these cells are not known, although mouse cell lines with a ligand profile similar to that found on mouse primary tumors (and human melanoma metastases) could be used to dissect the contribution of specific receptor-ligand interactions in melanoma recognition in models of transplantable melanoma relevant to the human disease. Therefore, we screened 4 mouse melanoma cell lines, including B16 and its variants B16F0 and B16F10, as well as 1 cell line established from RET transgenic mice. All melanoma cell lines bound the NKp46-Fc fusion protein, suggesting that they express ligands for NKp46 (Figure 2B). Only B16 cells exhibited clear binding of the NKG2D-Fc fusion protein, which was due to the expression of Rae- 1 and, to a lesser extent, murine ULBP-like transcript-1 (MULT-1; data not shown). The DNAM-1 ligands CD155 and CD112 were found on all cell lines, although the latter was found at consistently lower levels (Figure 2B). Furthermore, $\mathrm{H}-2 \mathrm{~K}^{\mathrm{b}}$ and $\mathrm{H}-2 \mathrm{D}^{\mathrm{b}}$ antigens were not detected on all melanoma cell lines, although in some experiments very low levels of MHC class I antigens were detected on the B16 cell line only. Lastly, CD48, ICAM-1, and CD40, which are ligands for 2B4, LFA-1, and CD40L, respectively, as well as the CD28 ligands CD80 and CD86 were not detected (Figure 2B and data not shown). Control C57BL/6 (B6) splenocytes were positive for $\mathrm{H}-2 \mathrm{~K}^{\mathrm{b}}, \mathrm{H}-2 \mathrm{D}^{\mathrm{b}}, \mathrm{CD} 48$, and CD54, whereas control YAC-1 lymphoma cells were positive for NKp46, NKG2D, and DNAM-1 ligands (data not shown). These data show 
A

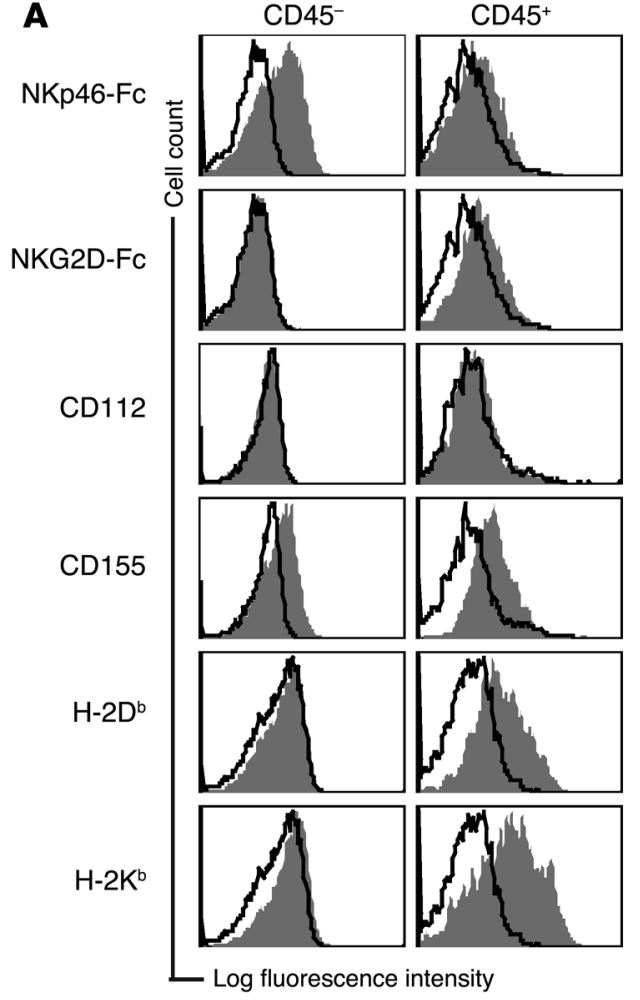

B

NKp46-Fc

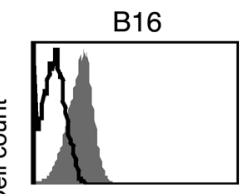

NKG2D-Fc

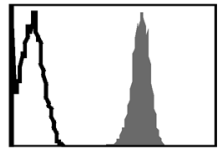

CD112

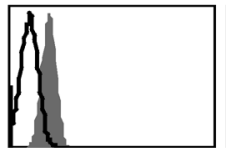

CD155

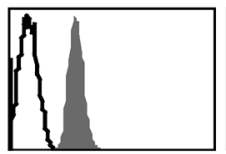

B16F0

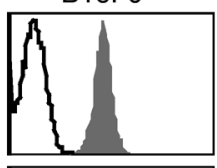

B16F10
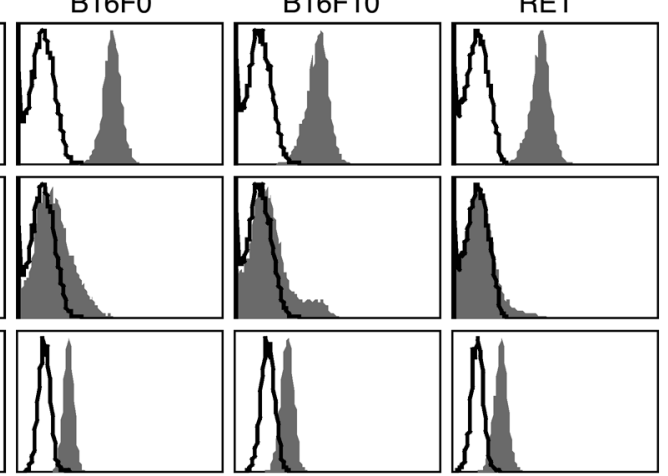

$\mathrm{H}-2 \mathrm{D}^{\mathrm{b}}$
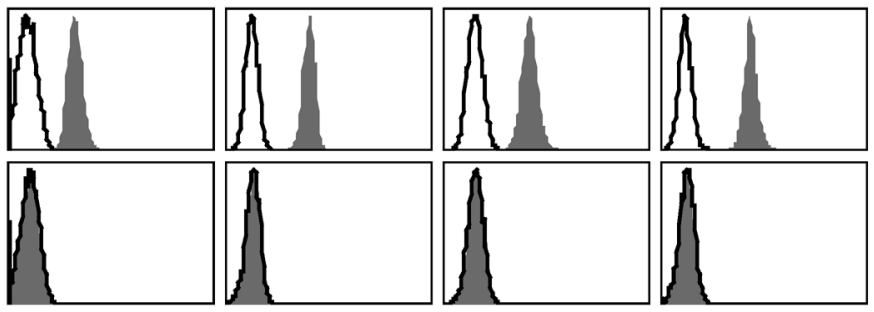

$\mathrm{H}-2 \mathrm{~K}^{\mathrm{b}}$
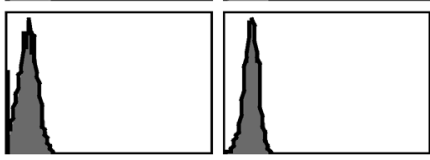

CD48
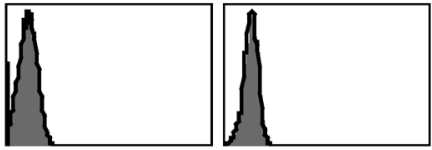

CD54
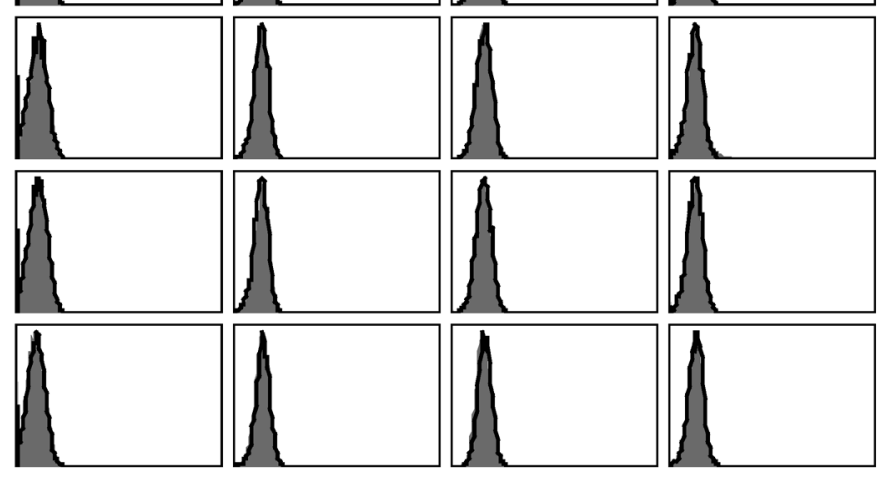

Log fluorescence intensity

\section{Figure 2}

Expression of ligands for NKp46 and DNAM-1 on murine melanoma cells. (A) Expression of the indicated ligands (filled histograms) relative to background staining determined by isotype-matched controls (open histograms) on single-cell suspensions prepared from tumor lesions freshly explanted from RET transgenic mice. CD45 expression was used to discriminate tumor infiltrating lymphocytes (CD45+) from the melanoma cells (CD45-). Histograms are representative of 4 individual mice that displayed similar stages of disease progression as judged by tumor size. (B) Expression of the indicated ligands (filled histograms) relative to background staining determined by isotype-matched controls (open histograms) on melanoma cell lines. Histograms are representative of 2-5 independent experiments.

that RET, B16F0, and B16F10 cell lines express ligands for the NK cell-activating receptors NKp46 and DNAM-1, thus resembling the ligand expression profile of mouse primary melanomas as well as human cell lines derived from LN melanoma metastases. Therefore, these cell lines are useful for studying the molecular interactions underlying the recognition of melanoma cells in vivo by NK cells. A comparative summary of ligand expression on melanoma cells in the 2 species is shown in Supplemental Table 1.

NCR and DNAM-1 mediate melanoma recognition in vitro in bumans and mice. Having established that NCR and DNAM-1 ligands are expressed on both human and mouse melanoma cells and that the counterreceptors are expressed on $60 \%-80 \%$ of human and $40 \%-60 \%$ of mouse NK cells (Supplemental Figure 4), we tested the functional relevance of these receptor-ligand interactions in NK cell recognition of melanoma cells in both species. First, we measured the effect of disrupting one or more of the receptorligand interactions in cell-mediated cytotoxicity using activated human allogeneic effector cells and blocking antibodies against individual receptors (the data on individual NCRs are not shown) or against multiple receptors simultaneously. We included blocking IgM antibodies against NKp30, NKp44, and NKp46 and blocking IgG antibodies against DNAM-1 and NKG2D because some of the human metastatic cell lines did show low expression of certain NKG2D ligands. Paired melanoma cell lines derived from LN and skin metastases of 2 patients (Mel16 and Mel1) were used as targets in order to compare the effect of antibody blockade with ligand expression in metastases from different anatomical sites. Only the simultaneous blockade of all NCRs and DNAM-1 significantly reduced cytotoxicity of metastatic LN cell lines $(P<0.002$ over the other conditions, except for the simultaneous blocking of NKG2D and DNAM-1; $P=0.07$ ) and showed a trend toward reducing cytotoxicity of SK cell lines (Figure 3A and data not shown). Additional NKG2D blocking did not reduce cytotoxicity further. Thus, NCR ligands may be expressed not only on LNs, but also 
A

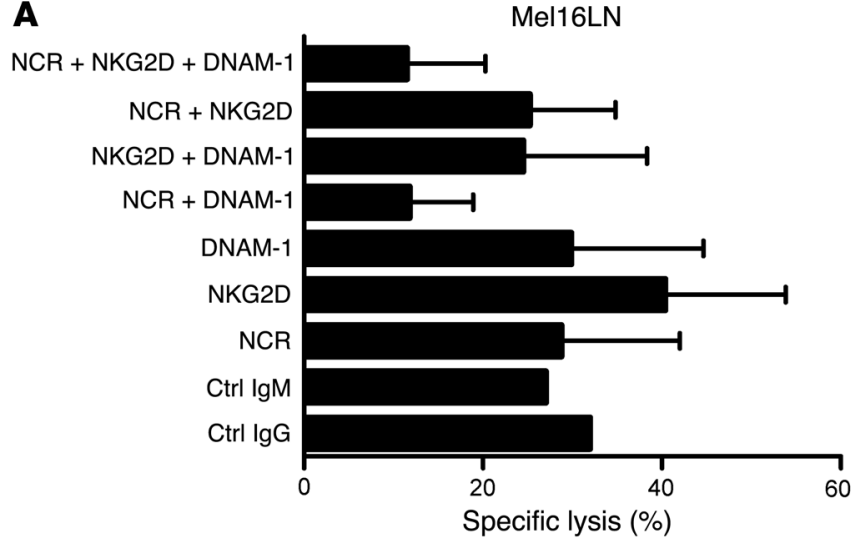

Mel16SK

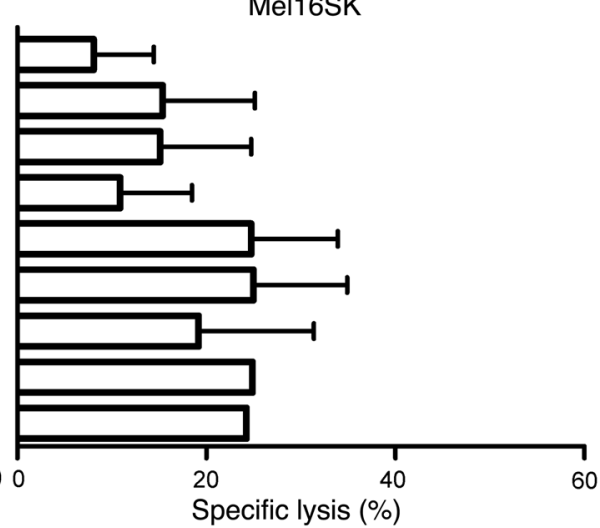

B

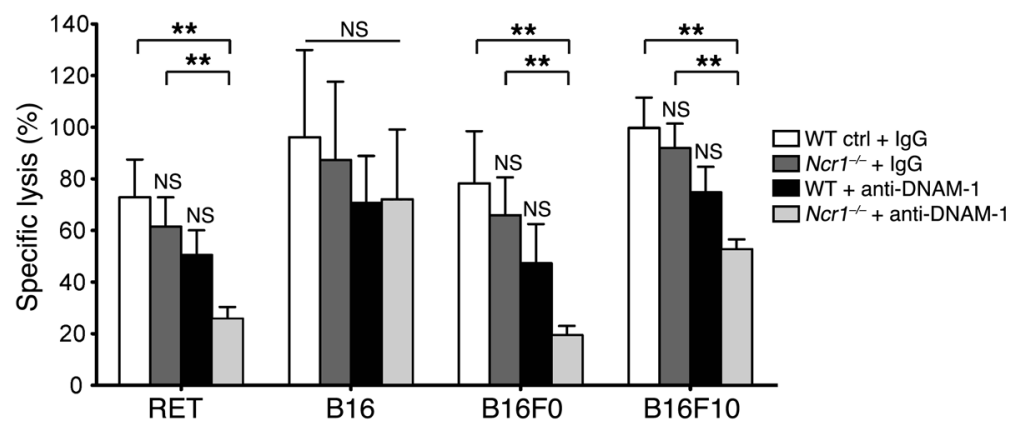

Figure 3

Interference with NCRs and DNAM-1 reduces melanoma recognition. (A) Allogeneic IL-2-activated NK cells from healthy donors were incubated at a 20:1 E:T ratio in the presence of the indicated combinations of the blocking monoclonal antibody, with tumor cells derived from paired melanoma cell lines of LN and skin metastases obtained from patient Mel16 and subjected to cytotoxicity assays. Cells incubated with control (Ctrl) IgG and control IgM antibodies were used as isotype controls. Data are mean + SD of 6 independent experiments, of which 4 included a control sample in which no monoclonal antibody was added (data not shown) and 2 included control IgG or IgM monoclonal antibody. (B) IL-2activated WT and $\mathrm{Ncr1}^{-1-} \mathrm{NK}$ cells were co-incubated with the indicated melanoma cells at a 20:1 E:T ratio in the presence of control IgG or anti-DNAM-1 monoclonal antibody and subjected to cytotoxicity assays. Data are the mean + SEM of 3 WT and 4 Ncr1 ${ }^{-/-}$mice and are pooled from 3 independent experiments. ${ }^{* *} P<0.01$ relative to WT cells in the presence of control IgG in that group.

on skin metastases, although they are undetectable with the Fc proteins (Figure 1A). This experiment also showed that melanoma cell lines derived from LN metastases of patients Mel16 and Mel1 were more susceptible to NK cell-mediated lysis compared with paired skin metastases of the same patient (Figure 3A and data not shown), suggesting that NK cells preferentially recognize and target LN metastases.

Similar results were obtained in blocking experiments carried out using IL-2-activated mouse NK cells and mouse melanoma cell lines. In this system we could take advantage of $\mathrm{Ncr}^{-/-}$mice. In the presence of control antibodies, WT and $\mathrm{Ncr}^{1^{-1-}} \mathrm{NK}$ cells killed melanoma cells (including RET, B16, B16F0, and B16F10) to a similar extent, although there was a slight reduction in cytotoxicity in $\mathrm{Ncr}^{-/-} \mathrm{NK}$ cells across the different cell lines (Figure 3B). A consistent, yet minor, reduction in cytotoxicity across the various cell lines was also caused by blockade with anti-DNAM-1 antibodies in WT cells (Figure 3B). However, in both cases the reduction was not significant, suggesting that blocking single receptors does not significantly interfere with melanoma cell recognition by IL-2activated NK cells. By contrast, interfering with both pathways simultaneously, by blocking DNAM-1 receptors on $\mathrm{Ncr}^{-/-}$cells, resulted in a marked and significant reduction of melanoma cell killing, including RET, B16F0, and B16F10 targets (the reduc- tion ranging from $45 \%$ to $71 \%$ ), but not B16 cells, presumably because they express high levels of NKG2D ligands, which may trigger compensatory signals. These results suggest that NCR and DNAM-1 ligands play a major role in NK cell-mediated recognition of melanoma cells in both species and that a degree of redundancy exists among NKp46 and DNAM-1 receptor signaling, with respect to the recognition and subsequent killing of melanoma cells in vitro.

$N K$ cells target human melanoma metastases from $L N$ more effectively than metastases from other anatomical sites. The data in Figure 3A suggest that LN metastases are more susceptible to NK cell-mediated lysis than their skin counterparts. To directly test this hypothesis, we measured the susceptibility to allogeneic NK cell-mediated lysis of 2 pairs of LN and skin metastatic cell lines derived from 2 melanoma patients (Mel14 and Mel16). Cell lines of both tissues and from both patients exhibited some degree of susceptibility to NK cell-mediated lysis, but the cell lines derived from LN metastases of both Mel14 and Mel16 were killed more effectively than those derived from skin metastases (Figure 4, A and B). Similar data were obtained using paired LN and SK lines from patient Mel1. Statistical analysis of a larger panel of samples including 7 unpaired LN and 6 skin metastases from 10 patients revealed that the differences in susceptibility to lysis was significant at the effector/target (E:T) 

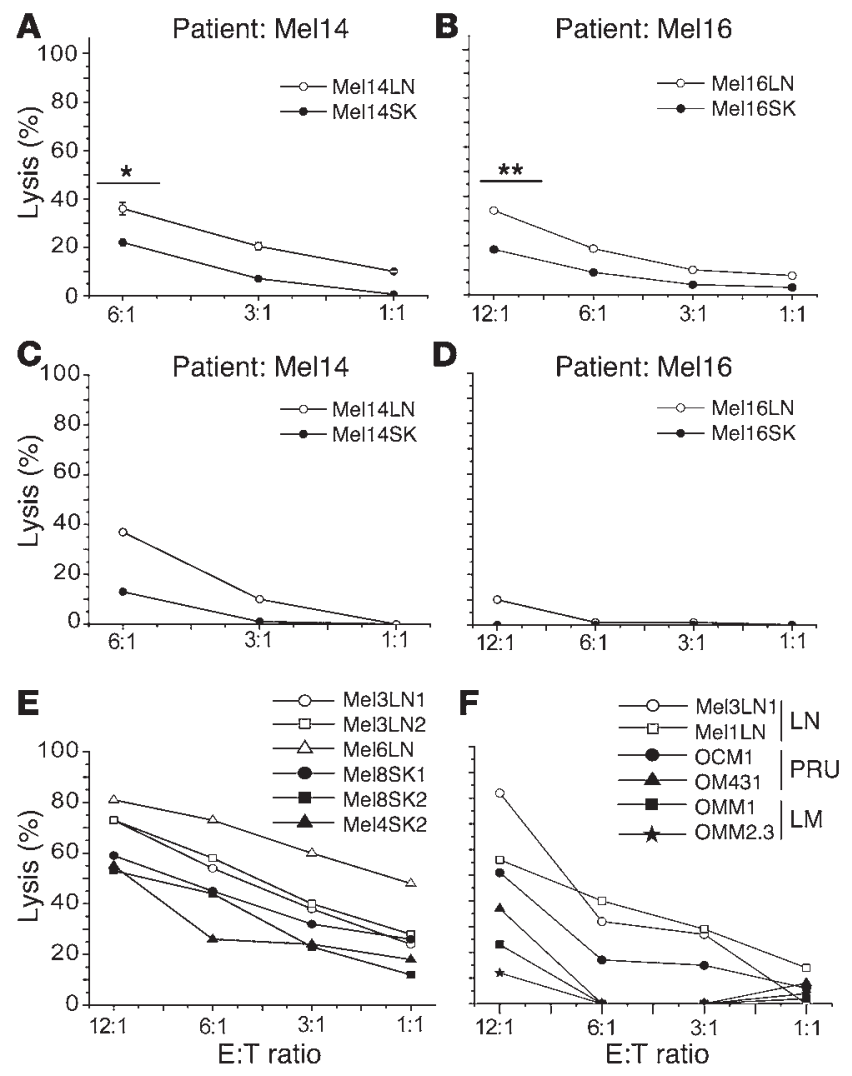

ratios tested (Supplemental Figure 5A). Cell lines derived from ascite (Mel10AS and Mel2AS) and pleura (Mel1PL and Mel2PL) metastases also showed lower susceptibility to NK cell recognition than did LN-derived cell lines (data not shown), suggesting that the susceptibility of LN metastases to NK cell-mediated killing is greater than that of metastases from various anatomical sites.

We then asked whether autologous NK cells also preferentially target LN metastases. To address this, we measured lysis by autologous NK cells of the 2 pairs of LN and skin metastases generated from patients Mel14 and Mel16. The data in Figure 4, C and D, clearly show that autologous NK cells also preferentially targeted LN metastases in both patients, suggesting that the melanoma patient's NK cells did retain the potential to discriminate between metastases of different anatomical origin. Similar data were obtained using paired LN and SK lines from patient Mel1. The data obtained with patient Mel16 (Figure 4D) indicate that lysis mediated by autologous NK cells may be less potent than that mediated by allogeneic NK cells (Figure 4C). This phenomenon may be explained on the basis of the engagement of inhibitory receptors by the self HLA class I antigens expressed, although at low level on tumor cells. Indeed, antibody blockade of HLA class I molecules did enhance the killing of autologous targets (data not shown).

In another experimental setting, we asked whether polyclonal resting allogeneic NK cells were also capable of preferential recognition of LN metastases compared with skin metastases. We used unpaired cell lines derived from 3 LN (Mel3LN1, Mel3LN2, and Mel6LN) and 3 skin (Mel8SK1, Mel8SK2, and Mel4SK2) metastases from 4 melanoma patients. Allogeneic NK cells killed LN metastatic cell lines more effectively than skin metastatic cell lines (Figure 4E). IL-2 slightly increased the NK cell-mediated cytotoxicity

\section{Figure 4}

LN metastases are preferentially recognized by NK cells. (A and B) Resting allogeneic NK cells from healthy donors were co-incubated with paired melanoma cell lines from $L N$ and skin metastases obtained from patients Mel14 and Mel16 and subjected to cytotoxicity assays. Data are mean \pm SEM of results from 6 experiments. ${ }^{*} P<0.05$, ${ }^{\star \star} P<0.005$ by Student's $t$ test. (C and D) Autologous NK cells were co-incubated with paired melanoma cell lines from LN and skin metastases obtained from patients Mel14 and Mel16 and subjected to cytotoxicity assays. Data are from 1 experiment performed. (E) Resting allogeneic NK cells from healthy donors were co-incubated with unpaired melanoma cell lines from LN (open symbols) and skin (filled symbols) metastases obtained from different patients and subjected to cytotoxicity assays. Data are representative of 1 of 4 experiments. (F) Resting allogeneic NK cells from healthy donors were co-incubated with melanoma cell lines from LN metastases, primary uveal melanoma (PRU), and uveal melanoma liver metastases (LMs) and subjected to cytotoxicity assays. Data are representative of 1 of 3 experiments.

but did not alter the preferential recognition of LN metastatic cell lines (data not shown).

To test whether the difference in susceptibility to NK cell recognition correlated with the route of metastasis progression, we compared the susceptibility of LN metastases with that of liver metastases obtained from patients with uveal melanoma. Uveal melanoma progresses exclusively through the hematogenous route due to the lack of a lymphatic system inside the eyes, and tumor spread is controlled mainly by NK cells (23). NK cells killed uveal melanoma-derived liver metastatic cell lines OMM1 and OMM2.3 less efficiently than cutaneous melanoma-derived LN metastatic cell lines Mel3LN1 and Mel1LN (Figure 4F). While the primary uveal melanoma cell lines OM431 and OCM1 were less susceptible to NK cell-mediated lysis than LN metastases, they were killed by NK cells nevertheless, suggesting that uveal melanoma cells are not intrinsically resistant to NK cell mediated killing (Figure 4F). Analysis of data obtained from 3 experiments showed a significant difference in the susceptibility of LN metastasis and uveal melanoma liver metastasis to lysis mediated by NK cells at lower E:T ratios $(P<0.05)$ (Supplemental Figure 5B). Collectively, these data suggest that NK cells control lymphatic dissemination more effectively than hematogenous dissemination. These data show that, irrespective of the genetic makeup of the donor and the patient, NK cells have an intrinsic capacity to discriminate between LN metastases and those from other anatomical sites and preferentially target LN metastases over those progressing through the blood. It therefore appears that the anatomical site and the route of dissemination set the level of susceptibility to NK cell lysis.

NK cells control murine melanoma tumor growth in vivo. We next used several in vivo models to examine the role of NK cells in the control of melanoma tumor growth. For this we employed mouse melanoma cell lines and predominantly a cell line derived from a facial nodule developed in a RET mouse (24). This RET cell line expresses a set of ligands similar to those expressed by primary melanomas (Figure 2B) and human metastases (Figure 1, A and B) and is susceptible to NK cell killing (Supplemental Figure 6). In a model of s.c. tumor growth in which mice had been depleted of NK $1.1^{+}$cells, we observed a protective effect of NK1.1 $1^{+}$cells at low melanoma cell doses (Figure 5A). Tumor growth was both substantially accelerated and increased in NK1.1-depleted mice compared with control mice, demonstrating that NK and/or NKT cells contribute to the resistance against RET melanoma cells. Similar 

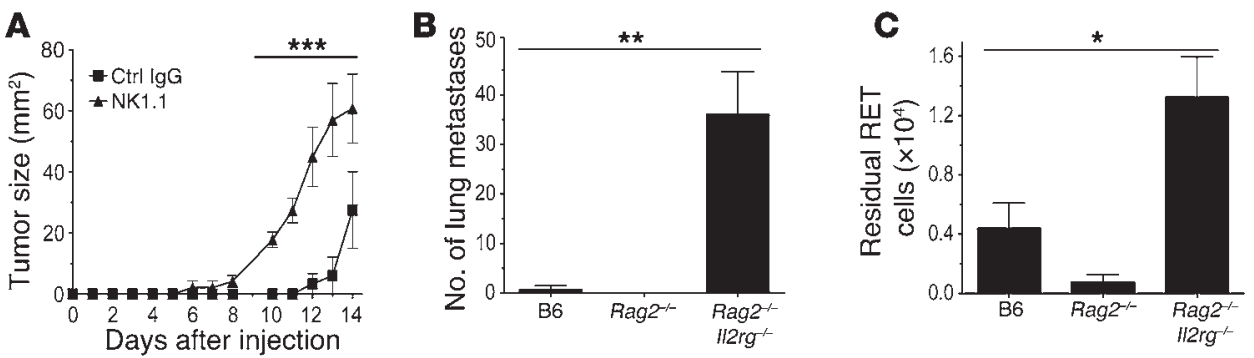

\section{Figure 5}

Innate NK cell immunity to melanoma cells in vivo. $(\mathbf{A})$ Cells $\left(1 \times 10^{4}\right)$ were injected s.c. into control IgG-treated or NK1.1-depleted syngeneic B6 mice. Tumor growth was monitored daily until mice were killed at day 14 . Data are mean \pm SEM of 5 mice per group. ${ }^{* * *} P<0.01 \mathrm{NK} 1.1 \mathrm{com}-$

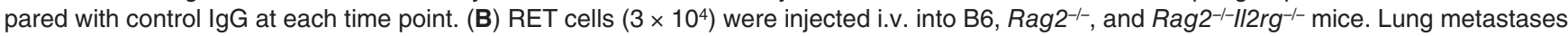
were counted 14 days later. Results are representative of 3 independent experiments. Data are mean + SEM of 4 mice per group. ${ }^{* \star} P<0.001$

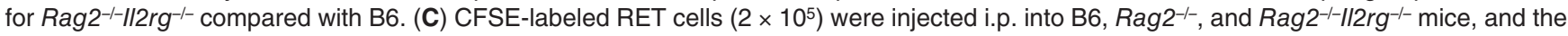
number of residual RET cells in the peritoneal cavity were counted following lavage 48 hours later. Results are representative of 4 independent experiments. Data are mean + SEM of 5 mice per group. ${ }^{*} P<0.05$ for Rag2 $^{-1-} / 12 \mathrm{rg}^{-/-}$compared with B6.

data were obtained using B16 and B16F10 cells (data not shown), in agreement with previous work (7).

To uncouple the role of NK cells from that of NKT cells, we used the well-established and clinically relevant model of lung metastases. We inoculated RET cells $\left(3 \times 10^{4}\right)$ i.v. into WT B6 mice and into

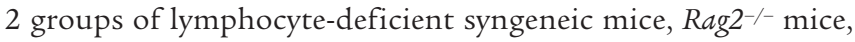
which lack B, T, and NKT cells but have NK cells, and Rag2-/-Il2 $\mathrm{rg}^{-1}$ mice, which lack all lymphocytes, including B, T, NKT, and NK cells. Metastases were hardly detectable in $\mathrm{B} 6$ and $\mathrm{Rag}^{-/-}$mice but were numerous in Rag2 ${ }^{-1-} \mathrm{Il} 2 \mathrm{rg}^{-1-}$ mice (Figure 5B). Similarly, high numbers of lung metastases were found in NK1.1-depleted B6 mice (data not shown).

To further uncouple the role of NK cells from that of NKT cells in acute recognition and rejection of melanoma, we used a short-term (2-day) assay based on i.p. tumor clearance. CFSE-labeled RET cells $\left(2 \times 10^{5}\right)$ were injected into the peritoneal cavity, and the residual tumor cells were enumerated 48 hours later in peritoneal lavages. Rag2-/-Il2 $\mathrm{rg}^{-/-}$mice had 3- to 10-fold more residual tumor cells than Rag2 ${ }^{-/-}$mice, which cleared tumor cells as effectively, if not more so, than WT mice (Figure 5C). Similarly high numbers of residual tumor cells were found in NK1.1-depleted B6 mice (data not shown). Together, the results from these in vivo experiments indicate that NK cells are necessary to acutely clear melanoma cells and prevent both melanoma growth and progression to metastasis.

NCR and DNAM-1 mediate melanoma recognition in vivo. Having confirmed that NK cells are crucial players in immunosurveillance of melanoma, and having established that this is also true in the context of the expression of a defined set of ligands on the melanoma cell lines, we anticipated that interrupting the NKp46 and DNAM-1 interactions with their ligands on melanoma cells would lead to reduced tumor clearance in vivo. To test this, we used 2 models of tumor clearance. The peritoneal clearance assay was used to model acute recognition of melanoma, whereas the lung metastasis assay was used to recapitulate the hematogenous spread of the disease. Mice were pretreated with anti-DNAM-1, control IgG, or anti-NK1.1 antibodies. Anti-DNAM-1 TX42 treatment did not deplete NK cells (confirming the data in ref. 20). Thus, NK cell percentages in the spleens of mice subjected to the peritoneal clearance assays were: IgG-treated mice, $2.89 \pm 0.85$, $n=4$; anti-DNAM-1 TX42-treated mice, $3.37 \pm 0.97, n=4$; antiNK1.1 PK136 monoclonal antibody-treated mice, $0.64 \pm 0.08$, $n=5$. NK cell percentages in the spleens of mice subjected to the lung metastases assays were: IgG-treated mice, $2.88 \pm 0.82$, $n=5$; TX42-treated mice, $2.3 \pm 0.52, n=5$; PK136-treated mice: $0.01 \pm 0.005, n=5$. Blocking the DNAM- 1 receptor strongly inhibited acute clearance of melanoma cells $(P=0.03$ versus mice treated with control IgG; Figure 6, A and B) to almost the same inhibition of clearance as in NK1.1-depleted mice, suggesting that acute recognition and rejection of melanoma cells in vivo is mediated chiefly via the DNAM-1 pathway, with little contribution from other pathways. In vitro, DNAM-1+ $\mathrm{NK}$ cells were much more potent killers of melanoma cells than DNAM-1- NK cells (Figure 6C). The 2 subsets killed lymphoma cells, though the DNAM-1- NK cells were less efficient. The different cytotoxic potential of the 2 subsets was not due to obvious differentiation disparity between DNAM- $1^{+}$and DNAM-1- NK cells because the pattern of CD11b and CD27 expression was indistinguishable between the 2 subsets. Moreover, both DNAM-1 ${ }^{+}$and DNAM-1- NK cells were NKp46 ${ }^{+}$ and NKG2D ${ }^{+}$(Supplemental Figure 7). DNAM- $1^{+}$is expressed in cells other than NK cells, and DNAM- 1 expression in $\mathrm{CD}^{+} \mathrm{T}$ cells and NK cells is important for lymphoma clearance, though the $T$ cell contribution is more marked in a s.c. model of tumor rejection (20). Thus, in order to assess the contribution of DNAM-1 on NK cell-mediated immunosurveillance, we used a variant of the peritoneal clearance assay, in which purified DNAM-1 ${ }^{+}$or DNAM-1NK cells isolated from WT or $\mathrm{Ncr}^{-/-}$mice were activated in IL-2 in vitro and used to clear melanoma cells administered in the peritoneal cavity of $\mathrm{Rag}^{-/-} \mathrm{Il2} \mathrm{rg}^{-/-}$mice. Both DNAM-1 ${ }^{+} \mathrm{NK}$ cells and DNAM-1- NK cells from WT and $\mathrm{Ncr}^{-1^{-1}}$ mice cleared most melanoma cells, but clearance by DNAM- $1^{+}$cells was greater (Figure 6D), supporting the notion that DNAM-1 is involved in acute recognition and rejection of melanoma. Interestingly, DNAM- $1^{+}$ NK cells were underrepresented in mouse primary melanomas $(11.9 \% \pm 6.3 \%, n=3)$ compared with splenic DNAM- ${ }^{+}$NK cells (40\%-60\%), suggesting that it could be beneficial to increase the number of DNAM- $1^{+} \mathrm{NK}$ cells at the site of tumor growth.

The contribution of NKp46 and DNAM-1 receptors in controlling lung metastases over 14 days was different from their contribution to acute melanoma rejection. The numbers of metastases was generally higher in $\mathrm{Ncr1}^{-/-}$mice than in WT mice (Figure 6E), and blocking DNAM-1 did not significantly increase the number of metastases, suggesting that NKp46, and not DNAM-1, may pre- 
A

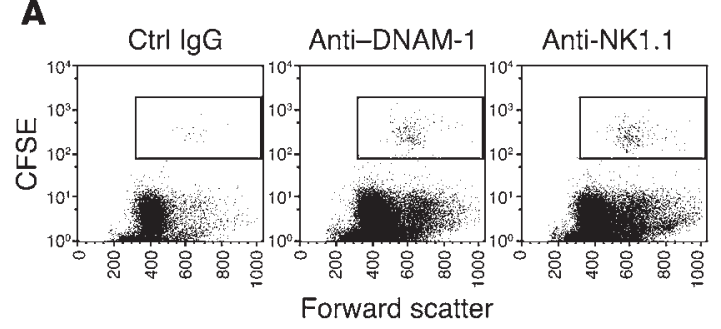

$\mathbf{C}$

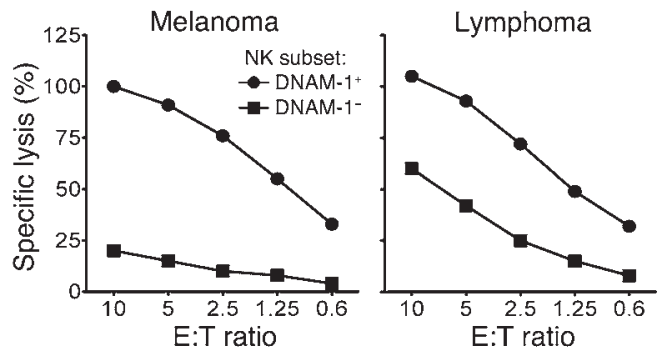

E

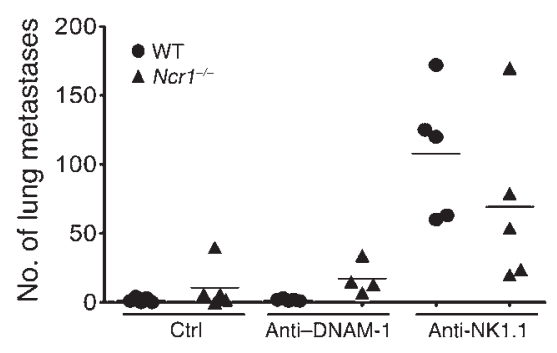

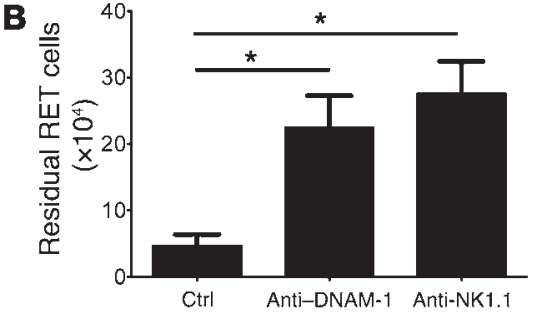

D

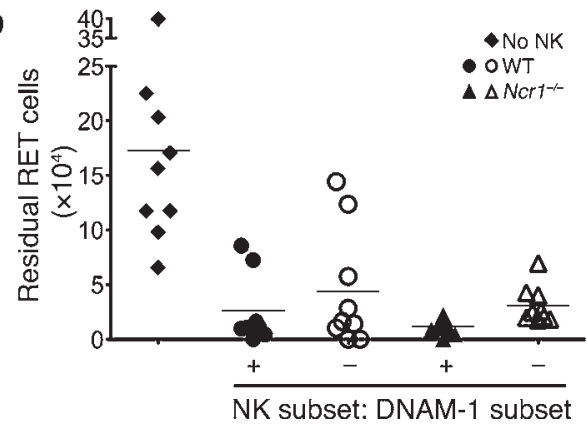

$\mathbf{F}$

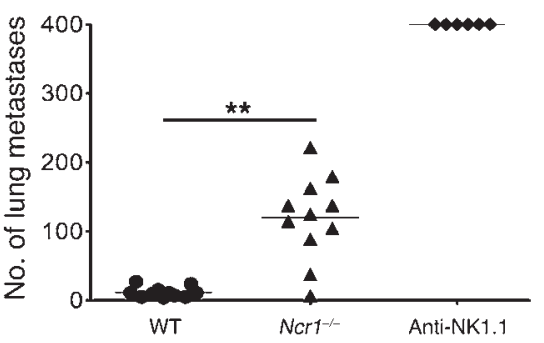

Figure 6

Differential contribution of NKp46 and DNAM-1. (A) CFSE-labeled RET cells $\left(2 \times 10^{5}\right)$ were injected i.p. into control IgG- and DNAM-1-treated or NK1.1-depleted B6 mice, and the number of residual RET cells were counted 48 hours later. Representative plots of peritoneal lavage CFSE gating are shown. (B) Quantification of residual cells. Data are mean + SEM of 8-10 mice per group and are pooled from 2 experiments. ${ }^{\star} P<0.05$ compared with control. (C) NK cells were expanded for 5 days in IL-2, sorted into DNAM-1+ and DNAM-1-, recultured for 48 hours, and tested for cytotoxicity at the indicated E:T ratio. Results are representative of 2-3 experiments. (D) NK cells from WT or Ncr1 ${ }^{-1-}$ mice were expanded, sorted,

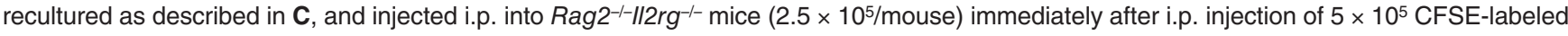
RET cells. Residual RET cells were counted following lavage 48 hours later. Results are individual mice from 3 independent experiments. The bar represents the mean of 8-9 mice per group. (E and F) RET cells $\left(10^{5}\right)$ were injected i.v. into control IgG, anti-DNAM-1-treated or anti-NK1.1depleted WT or $\mathrm{Ncr}^{-1-}$ mice (E). (F) RET cells $\left(2 \times 10^{5}\right)$ were injected i.v. into WT, Ncr1-1-, or NK1.1-depleted WT mice. Lung metastases were counted 14 days later. Results are from individual mice, and the bar represents the mean of $4-5(E)$ and $6-11(F)$ mice per group. ${ }^{\star \star} P<0.01$.

vent lung metastases. The key role of NKp46 in preventing lung metastases was confirmed in a second experiment in which we administered twice as many melanoma cells. (Figure 6F). In both cases, NK1.1-depleted mice had greater numbers of lung metastases than $\mathrm{Ncr}^{-/-}$mice, suggesting that other receptors on NK1.1 $1^{+}$ cells are involved in melanoma cell recognition. These results confirm that NK1.1+ cells are essential to contain lung metastases, suggest that NKp46, but not DNAM-1, is critical to controlling the hematogenous spread of melanoma to viscera and that other receptors may also contribute to this control, and highlight the impact of the anatomical site in determining the NK cell receptors that play critical roles in immunosurveillance in vivo.

NK cells control human melanoma tumor growth in vivo. Based on the in vitro evidence that NK cell-mediated recognition of melanoma metastatic cell lines is influenced by the anatomical origin of the metastases (Figure 4 and Supplemental Figure 5, A and B), we tested the relevance of this finding in vivo. To this end, SCID mice were infused with melanoma cell lines derived from skin, LN, pleura, or liver metastases. Adoptively transferred human NK cells were then tested for their ability to control melanoma tumor growth. The mice developed advanced disease in 10-11 days in the bone marrow, and mice that had not received any NK cells died within 1 month (Figure 7A). In contrast, mice infused with human NK cells $\left(5 \times 10^{6}, 3 \times 10^{6}\right.$, or $1.5 \times 10^{6} /$ mouse; Figure $7 \mathrm{~A}$ and data not shown) controlled melanoma tumor growth irrespective of the metastasis origin of the infused cell line and survived without melanoma infiltration. When low numbers of NK cells $\left(5 \times 10^{5}\right.$ cells/mouse $)$ were infused, mice transplanted with skin, pleura, and liver metastatic cell lines died, while mice transplanted with LN metastatic cell lines survived without melanoma infiltration (Figure 7B). These data demonstrate that LN metastatic melanoma cell lines are more susceptible to NK cell-mediated lysis in vivo than melanoma cell lines derived from metastases in other anatomic sites. 

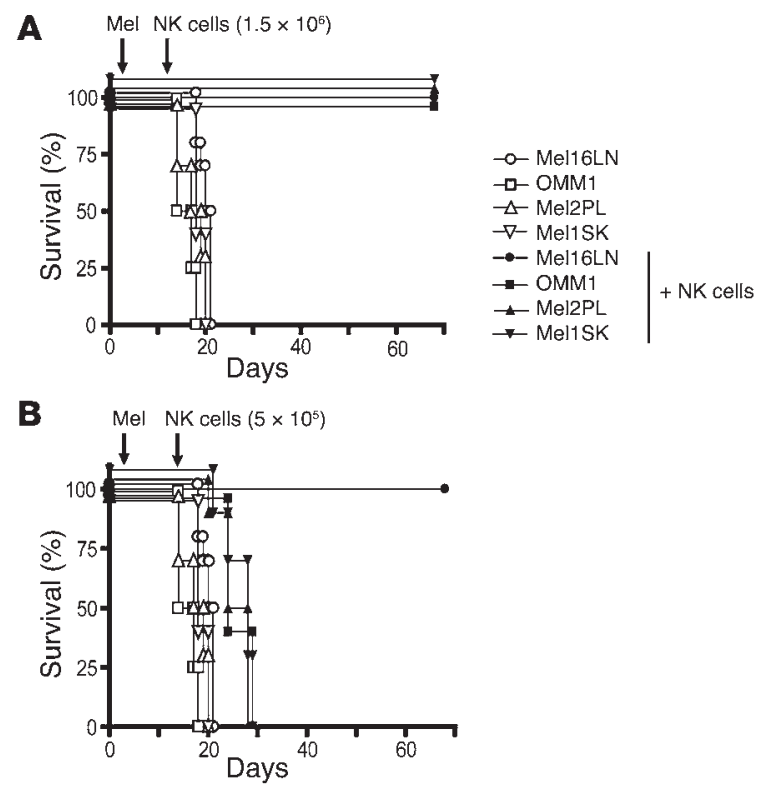

\section{Discussion}

One of the obstacles to the advancement of current melanoma immunotherapy is the limited understanding of the molecular mechanisms of immunological recognition. Our data show, for what we believe is the first time, that malignant melanoma cells express a set of ligands that mediate NK cell recognition and that are shared among human metastases, murine primary melanomas, and murine cell lines. Recognition of these ligands by the NK cellactivating receptors NCRs and DNAM-1 mediates melanoma cell killing. This has major implications for developing new melanoma treatments, as identifying the ligand/receptor pathways that cooperate in vivo is critical for future NK cell-based immunotherapy. While it is possible that other, undefined ligands for activating receptors are present on melanoma cells, our study shows that the functional interactions between NCRs and DNAM-1 and their ligands are biologically relevant, as disruption of these interactions blocks NK cell cytotoxicity and impairs melanoma rejection. Nonetheless, other receptors are likely to be implicated in the recognition of melanoma.

The contribution of NCRs and DNAM-1 to killing of melanoma cells may vary depending on the activation status of the NK cells, the anatomical site of the tumor, and the route of metastatic progression. Clearly, some level of additive cooperation between receptors is required for in vitro killing of melanoma cells in both mice and humans. Conversely, the 2 receptors appear to have divergent roles in acute recognition of melanoma and control of metastases in mice. Acute recognition is DNAM-1 dependent but not NKp46 dependent, whereas control of lung metastases is NKp46 dependent but not DNAM-1 dependent. In addition to the temporal progression of malignancy, the spatial distribution of relevant ligands and NK receptors may account for our observation. The expression of DNAM-1 ligands is restricted to endothelial cells, placenta, and hematopoietic cells (25), and it will be important to define DNAM-1 expression on tissue-associated NK cells. Tissue-specific infiltration by $\mathrm{NK}$ cell populations expressing distinct receptor repertoires has been reported previously. Thus, NCR-expressing NK clones were required for the elimination of lung carcinoma and,

\section{Figure 7}

Higher susceptibility of LN metastases in a xenogeneic model of immunotherapy for melanoma. (A) NOD-SCID mice (3 mice/group) were engrafted with melanoma cell lines (mel) $\left(2 \times 10^{6}\right.$ cells/mouse) from LN, liver (OMM1), pleura, and skin in the presence (filled symbols) or absence (open symbols) of NK cells adoptively transferred from allogeneic healthy donors $\left(1.5 \times 10^{6}\right.$ cells/mouse $)$ on day 10 following the administration of melanoma cells. Mice were then monitored for survival up to day 70. (B) The same procedure was followed as described in $\mathbf{A}$, except that fewer NK cells $\left(5 \times 10^{5} \mathrm{NK}\right.$ cells/mouse) were adoptively transferred from allogeneic healthy donors on day 10 following the administration of melanoma cells. Mice were monitored for survival up to day 70 . The experiment was repeated twice with similar results.

conversely, loss of NCR expression on lung-resident NK cells led to tumor progression (26). Our data corroborate the observation that $\mathrm{NCR}^{+} \mathrm{NK}$ cells are crucial for antitumor activity in the lung.

NK cells are necessary to provide innate immunity to the outgrowth of transplanted melanoma. However, during spontaneous melanoma formation, other immune cells including NKT and $\mathrm{T}$ cells may also contribute to melanoma resistance. Notwithstanding the likely participation of other leukocytes to melanoma immunity, syngeneic mouse NK cells, autologous human NK cells, and allogeneic NK cells from healthy donors have the intrinsic capacity to recognize and target malignant melanoma cells. This has important implications for immunotherapy, as 2 nonexclusive scenarios may be envisaged to improve NK cell immunity in patients: augmentation of endogenous NK cell activation/migration pathways and intervention with cell therapy, thus implementing allogeneic NK cells (Figure 8). Allogeneic NK cells with killer cell immunoglobulin-like receptor incompatibility show enhanced cytotoxicity against melanoma cell lines (27), but this strategy remains to be tested in patients. In light of our results, augmenting DNAM-1-mediated recognition of melanoma appears an attractive approach. Mouse primary melanomas and human biopsies contained very few NK cells $(0.4 \% \pm 0.3 \%, n=3$ in cell suspensions from leukocytes infiltrating primary tumors), and DNAM- $1^{+}$cells were underrepresented $(11.9 \% \pm 6.3 \%, n=3)$ compared with splenic NK cells (40\%-60\%). Given the more efficient cytotoxic potential of DNAM- $1^{+} \mathrm{NK}$ cells, potentiating the number or migration of DNAM- $1^{+} \mathrm{NK}$ cells in situ may help combat melanoma.

Irrespective of their origin, all human metastatic cells expressed ligands for DNAM-1 and low levels of MHC class I molecules, rendering them susceptible to NK cell killing. However, the comparatively greater susceptibility of human LN metastases to NK cell recognition correlated with higher NCR ligand expression, and thus anatomical compartmentalization of ligand expression and expression of the ligands during disease progression may also contribute to the differential roles of NK cell receptors in melanoma recognition. For example, the LN metastases were reported to precede the appearance of skin metastasis in the 2 patients (Mel1 and Mel16) whose paired metastases were available for direct comparison. It is therefore tempting to speculate that in these patients NKp46 and NKp44 ligands were overexpressed in the early stages of the disease, as suggested by a recent study (28) and that tumor progression leads to melanoma cell variants that express low levels of NCR ligands. Our data are reminiscent of results obtained in a recent study, which indicated a marked reduction of NCR ligand expression on acute myeloid leukemia cells compared with normal myeloid cells (29). 
A

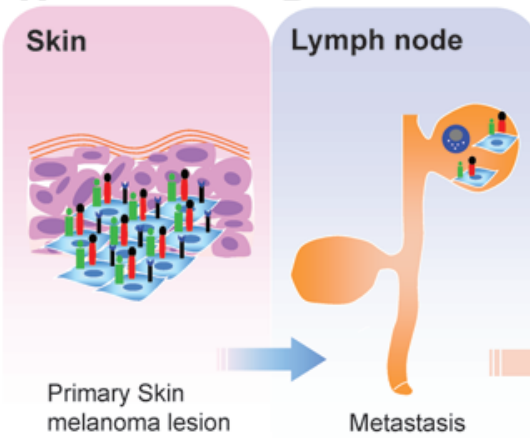

B

melanoma lesion
C

Blood

D

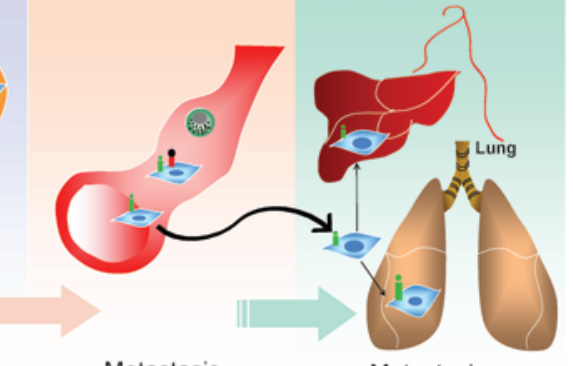

Editing??

Metastasis

Escape

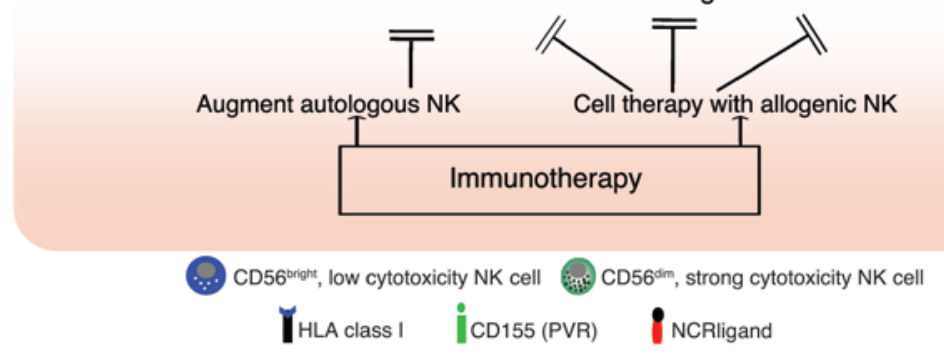

Figure 8

Receptor-mediated NK cell recognition of melanoma and opportunities for immunotherapy. (A) The early stages of melanoma cells are opposed by only rare resident or infiltrating NK cells, and these melanoma cells eventually metastasize to the draining LN. Although ligands for both DNAM-1 and NCRs are expressed, along with low expression of MHC class I molecules, targeting the DNAM-1 pathway might be an effective immunotherapeutic strategy at this stage, for example, by infusing autologous or allogeneic DNAM-1+ NK cells. (B) Once in the LN, the melanoma cells might undergo a weak and inefficient selection by the low cytotoxic CD56 bright NK cells. (C) Hematogenous spread of melanoma cells might undergo a stronger selection by the highly cytotoxic CD56 dim blood NK cells, resulting in an editing out of the melanoma cells expressing NCR ligands. (D) The melanoma cells not expressing NCR ligands escape NK cells and metastasize to visceral organs. Immunotherapeutic intervention at later stages of the disease based on the use of strong cytotoxic NK cells could be envisaged by either deliberately recruiting autologous NK cells from the patient's pool into the LN or by adoptively transferring allogeneic NK cells from healthy donors in an attempt to halt the metastatic progression to visceral organs. This oversimplified view of melanoma progression is placed in the context of NK cell recognition and does not take into account the interactions of melanoma cells with other immune cells.

How can we explain the differences in expression of NCR ligands on LN versus skin melanoma metastatic cells? Phenotypes associated with the different melanoma metastases may result from an NK-mediated immunoediting process or phenotypic modulation (e.g., by cytokines, interaction with stromal cells, etc.). Two testable scenarios may explain our data. Human NK cell populations can be divided into 2 discrete subsets $(30,31)$, CD56 $6^{\text {bright }}$ and CD56 dim, with the former, poorly cytotoxic subset present mainly in the $\mathrm{LN}$, while the latter, highly cytotoxic subset predominates in the periphery. Therefore, it is conceivable that LN CD56 $6^{\text {bright }} \mathrm{NK}$ cells do not edit LN metastases efficiently. Alternatively, LN NK cells do actually edit early melanoma metastatic cells that strongly express NKp44 and NKp46 ligands, but their number is insufficient to prevent disease progression. Whatever the immunosubversion mechanisms discussed above, our xenogeneic model of cell therapy showed that infusion of a low number of allogeneic NK cells eliminated LN metastases but did not affect metastases from skin, ascites, or pleura. In contrast, infusion of a high number of allo-

geneic NK cells did cure animals transplanted with any melanoma metastases, regardless of their origin.

Collectively, our data suggest that peripheral blood-derived allogeneic NK cells could target tumors infiltrating the $\mathrm{LN}$ at early stages of disease. We propose an intriguing scenario for the design of new anticancer immunotherapy approaches to prevent melanoma dissemination in its early stages, by either reprogramming the anatomical distribution of NK subsets using appropriate TLR ligands, as recently described in mice (32), or by adoptively transferring NK cells from healthy donors (Figure 8).

\section{Methods}

Mice. B6 RET mice were bred at the Babraham Institute, the Department of Microbiology, Tumor and Cell Biology (Karolinska Institutet), and the German Cancer Research Center. Rag2 $2^{-/-}, \mathrm{Rag}^{-/-} \mathrm{Il}_{\mathrm{rg}^{-/}}$, and $\mathrm{Ncr}^{-1-}$ mice (all on a B6 background) were bred at the Babraham Institute. $\mathrm{Ncr}^{-/-}$mice were a kind gift of O. Mandelboim (Hebrew University, Jerusalem, Israel). NOD-SCID mice were established at the Department of Clinical Medicine, University of Perugia. Experimental mice were used at 6-12 weeks of age. All procedures were approved by local ethical committees and were conducted in accordance with government and institute regulations (UK Home Office Project Licence 80/1908; Italian Ministry of Health, Department of Food, Nutrition and Animal Health decree no. 77/2007-B; Ethics Committee of the Heidelberg University 35-9185.81/G-41/03; The Stockholm Northern Animal Ethics Committee).

Cell lines and tumor lesions. All human melanoma cell lines were originated from metastatic lesions removed from patients with advanced-stage melanoma after informed consent, according to a previously described procedure (33) and with ethical permission from the Ethics Committee II of Heidelberg University (0198.3/2002). Patients were numbered Mel1-Mel18. Patients Mel1-Mel14 were melanoma patients from the Clinical Cooperation Unit for Dermato-Oncology, Mannheim, Germany. Patients Mel15-Mel18 were melanoma patients from the Fondazione IRCCS Istituto Nazionale dei Tumori, Milan, Italy. The human melanoma metastatic cell lines were kept in culture for 5-6 in vitro passages. The cells were used in different experimental settings within 2-3 in vitro passages. They were then discarded, since their phenotypes were changing after 4-5 weeks of in vitro culture. All tumors were HLA typed using serological and molecular methods. Haplotypes and clinical features of the patients are reported in Table 1. The primary human uveal melanoma cell lines OM431 (34) and OCM1 (35); the liver metastatic human uveal melanoma cell lines OMM1 (36) and OMM2.3 (37); and the B6-derived murine melanoma cell lines B16, B16F0, B16F10 and the MT/ret melanoma cells, a kind gift of A. Prevost-Blondel (Institut Cochin, Paris, France) (24) were all cultured in RPMI 1640 medium/5\% FCS/2 mM L-glutamine/50 $\mu \mathrm{M}$ 2-mercaptoethanol in a $5 \% \mathrm{CO}_{2}$ humidified atmosphere at $37^{\circ} \mathrm{C}$. All tumor cells were used for assays within 1 hour of collection, and viability was always greater than $95 \%$ as determined by Trypan blue exclusion. Normal human epidermal melanocytes from PromoCell were used in staining experiments (see Supplemental 
Methods). They were cultured using melanocyte growth medium M2 at $37^{\circ} \mathrm{C}$ in a humidified incubator with $5 \% \mathrm{CO}_{2}$.

Isolation of fresh tumor cells from RET mice. Fresh tumor samples were immediately transferred into serum-free RPMI 1640 medium and stored on ice. After removal of necrotic tissue and fat, tumors were cut into small pieces, filtered, washed, and analyzed by flow cytometry (Supplemental Methods).

Infusion of NK cells in buman melanoma cell-engrafted NOD-SCID mice. Peripheral blood mononuclear cells were depleted of $\mathrm{T}$ cells by negative anti-CD3 immunomagnetic selection (Miltenyi Biotec), activated with phytohemagglutinin (Biochrom KG), and cultured with IL-2 (Chiron, Novartis) as previously described (ref. 38 and Supplemental Methods). NK cells were screened for cytotoxicity by standard ${ }^{51} \mathrm{Cr}$ release assay against melanoma cells at a decreasing E:T ratio (20:1, 10:1, 5:1, and 2.5:1; Supplemental Methods). NOD-SCID mice (Charles River Laboratories) were irradiated ( $3.5 \mathrm{~Gy}$ ), followed by injection of $2 \times 10^{6}$ melanoma metastatic cell lines derived from pleura (Mel2PL), liver (OMM1), skin (Mel1SK), and LNs (Mel16LN) i.v. the next day. Once clinical signs of engraftment were observed (mean of 10 days), mice were sacrificed, and cell suspensions from bone marrow, spleen, LNs, lung, liver, and brain were evaluated for melanoma infiltration by flow cytometry using anti-human MHC class I monoclonal antibody (W6/32HL) and anti-Ig FITC labeled. At this time point, we infused NK cells. We divided each group of mice engrafted with LN, liver, skin, or pleura melanoma metastases into 5 subgroups ( 3 mice) subgroup). Subgroup 1 included controls that didn't receive NK cell treatment, subgroup 2 received $5 \times 10^{6} \mathrm{NK}$ cells, subgroup 3 received $3 \times 10^{6}$ NK cells, subgroup 4 received $1.5 \times 10^{6} \mathrm{NK}$ cells, and subgroup 5 received $5 \times 10^{5} \mathrm{NK}$ cells. Experiments were repeated twice. Four months after NK cell infusion, melanoma cells were not detectable in the surviving mice.

In vivo tumor models. Tumor cells were harvested and injected at the indicated numbers in the hind thigh (s.c. model), tail vein (lung metastasis model), or peritoneal cavity (peritoneal clearance assay). For the peritoneal assay, tumor cells were labeled with $5 \mu \mathrm{M}$ CFSE (Invitrogen) according to standard protocols prior to injection. Mice were culled 48 hours after tumor injection, and the contents of the peritoneal cavity were harvested by lavage. The percentage of $\mathrm{CFSE}^{+}$tumor cells was determined by flow cytometry, and the number was calculated with respect to the total volume of lavage fluid administered. In some mice, the indicated number of IL-2-expanded NK cells was also delivered i.p. at time of tumor injection (Figure 6D). For the s.c. outgrowth model, tumor growth was monitored daily thereafter and the size of the tumor measured with digital calipers. For the lung metastasis model, mice were culled 14 days after i.v. adminis-

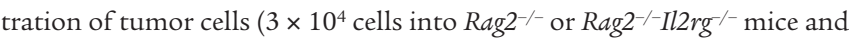
$1-2 \times 10^{5}$ cells into WT or and $\mathrm{Ncr}^{-/-}$mice) and the lungs fixed in Fekete's solution prior to enumeration of metastatic nodules. In some experiments, NK cells were first depleted by i.p. injection of $200 \mu \mathrm{g}$ anti-NK1.1 antibody (PK136, a gift of G. Butcher, The Babraham Institute) at day -1 (peritoneal assay) and days 3, 6, and 10 (lung and s.c. assays). Alternatively, DNAM-1 was blocked by administration of $200 \mu \mathrm{g}$ anti-CD226 antibody (TX42) i.p. on day -1, day 1 (peritoneal and lung assay), and days 6 and 10 (lung assay). Control mice received PBS or $200 \mu \mathrm{g}$ mouse IgG (Jackson ImmunoResearch Laboratories Inc.).

Statistics. Data obtained from multiple experiments are reported as mean \pm SEM. 2-tailed Student's $t$ test was used to determine statistical significance between control and test groups. A $P$ value of less than 0.05 was considered significant. ANOVA was performed to compare multiple human melanoma metastatic cell line groups, and if significant, a posthoc analysis was performed using Fisher's exact test with GraphPad Prism 4.0 and Statistica 6 software.

Note added in proof. During revision and production of our manuscript, 3 manuscripts have been published that show the importance of NKp46 and DNAM-1 in NK cell immunosurveillance in vivo (39-41).

\section{Acknowledgments}

The work was supported by grants from the Medical Research Council (to F. Colucci); the Associazione Italiana Ricerca sul Cancro (AIRC; to E. Carbone); the Swedish Cancer Society, the Swedish Research Council, and the Karolinska Institutet (to P. Höglund); and the Dr. Mildred Scheel Foundation for Cancer Research (106096, to V. Umansky and D. Schadendorf). We wish to thank Maureen Hamon and Amanda Hutchings for antibody production, Geoff Morgan for flow cytometry, the Babraham Small Animal Facility for mouse husbandry, and Daniel Hampshire for reading the manuscript, as well as members of the Carbone and Colucci labs for discussions.

Received for publication October 3, 2008, and accepted in revised form February 18, 2009.

Address correspondence to: Ennio Carbone, University of Catanzaro "Magna Graecia", Department of Experimental and Clinical Medicine, Tumor Immunology Laboratory, Viale Europa, 88100, Catanzaro, Italy. Phone: 39-0961-3694088; Fax: 39-0961-3694090; E-mail: ennio.carbone@ki.se. Or to: Francesco Colucci, Laboratory of Lymphocyte Signalling and Development, The Babraham Institute, CB22 3AT, Cambridge, United Kingdom. Phone: 44-1223-496612; Fax: 44-1223-496023; E-mail: francesco.colucci@bbsrc.ac.uk.
1. Walker, G.J., and Hayward, N.K. 2002. Pathways to melanoma development: lessons from the mouse. J. Invest. Dermatol. 119:783-792.

2. Chin, L. 2003. The genetics of malignant melanoma: lessons from mouse and man. Nat. Rev. Cancer. 3:559-570.

3. Recio, J.A., et al. 2002. Ink4a/arf deficiency promotes ultraviolet radiation-induced melanomagenesis. Cancer Res. 62:6724-6730.

4. Kato, M., et al. 1998. Transgenic mouse model for skin malignant melanoma. Oncogene. 17:1885-1888.

5. Kim, S., Iizuka, K., Aguila, H.L., Weissman, I.L., and Yokoyama, W.M. 2000. In vivo natural killer cell activities revealed by natural killer cell-deficient mice. Proc. Natl. Acad. Sci. U. S. A. 97:2731-2736.

6. Smyth, M.J., Hayakawa, Y., Takeda, K., and Yagita, H. 2002. New aspects of natural-killer-cell surveillance and therapy of cancer. Nat. Rev. Cancer. 2:850-861.

7. Smyth, M.J., Taniguchi, M., and Street, S.E. 2000. The anti-tumor activity of IL-12: mechanisms of innate immunity that are model and dose depen- dent. J. Immunol. 165:2665-2670.

8. Shankaran, V., et al. 2001. IFNgamma and lymphocytes prevent primary tumour development and shape tumour immunogenicity. Nature. 410:1107-1111.

9. Swann, J.B., and Smyth, M.J. 2007. Immune surveillance of tumors. J. Clin. Invest. 117:1137-1146.

10. Boon, T., Coulie, P.G., Van den Eynde, B.J., and van der Bruggen, P. 2006. Human T cell responses against melanoma. Annu. Rev. Immunol. 24:175-208.

11. Bancroft, G.J. 1993. The role of natural killer cells in innate resistance to infection. Curr. Opin. Immunol. 5:503-510.

12. Trinchieri, G. 1989. Biology of natural killer cells. Adv. Immunol. 47:187-376.

13. Colucci, F., Di Santo, J.P., and Leibson, P.J. 2002. Natural killer cell activation in mice and men: different triggers for similar weapons? Nat. Immunol. 3:807-813.

14. Lanier, L.L. 2005. NK cell recognition. Annu. Rev. Immunol. 23:225-274.
15. Bottino, C., et al. 2003. Identification of PVR (CD155) and Nectin-2 (CD112) as cell surface ligands for the human DNAM-1 (CD226) activating molecule. J. Exp. Med. 198:557-567.

16. Bryceson, Y.T., March, M.E., Ljunggren, H.G., and Long, E.O. 2006. Synergy among receptors on resting NK cells for the activation of natural cytotoxicity and cytokine secretion. Blood. 107:159-166.

17. Castriconi, R., et al. 2004. Natural killer cell-mediated killing of freshly isolated neuroblastoma cells: critical role of DNAX accessory molecule-1-poliovirus receptor interaction. Cancer Res. 64:9180-9184.

18. Carlsten, M., et al. 2007. DNAX accessory molecule-1 mediated recognition of freshly isolated ovarian carcinoma by resting natural killer cells. Cancer Res. 67:1317-1325.

19. El-Sherbiny, Y.M., et al. 2007. The requirement for DNAM-1, NKG2D, and NKp46 in the natural killer cell-mediated killing of myeloma cells. Cancer Res. 67:8444-8449.

20. Tahara-Hanaoka, S., et al. 2006. Tumor rejection 
by the poliovirus receptor family ligands of the DNAM-1 (CD226) receptor. Blood. 107:1491-1496.

21. Gazit, R., et al. 2006. Lethal influenza infection in the absence of the natural killer cell receptor gene Ncr1. Nat. Immunol. 7:517-523.

22. Cagnano, E., et al. 2007. Expression of ligands to NKp46 in benign and malignant melanocytes. J. Invest. Dermatol. 128:972-979.

23. Jager, M.J., Hurks, H.M., Levitskaya, J., and Kiessling, R. 2002. HLA expression in uveal melanoma: there is no rule without some exception. Hum. Immunol. 63:444-451.

24. Lengagne, R., et al. 2004. Spontaneous vitiligo in an animal model for human melanoma: role of tumorspecific CD8+ T cells. Cancer Res. 64:1496-1501.

25. Lopez, M., et al. 1998. The human poliovirus receptor related 2 protein is a new hematopoietic/ endothelial homophilic adhesion molecule. Blood. 92:4602-4611.

26. Le Maux Chansac, B., et al. 2005. NK cells infiltrating a MHC class I-deficient lung adenocarcinoma display impaired cytotoxic activity toward autologous tumor cells associated with altered NK celltriggering receptors. J. Immunol. 175:5790-5798.

27. Igarashi, T., et al. 2004. Enhanced cytotoxicity of allogeneic NK cells with killer immunoglobulin-like receptor ligand incompatibility against melanoma and renal cell carcinoma cells. Blood.
104:170-177.

28. Cagnano, E., et al. 2008. Expression of ligands to NKp46 in benign and malignant melanocytes. J. Invest. Dermatol. 128:972-979.

29. Nowbakht, P., et al. 2005. Ligands for natural killer cell-activating receptors are expressed upon the maturation of normal myelomonocytic cells but at low levels in acute myeloid leukemias. Blood. 105:3615-3622.

30. Fehniger, T.A., et al. 2003. CD56bright natural killer cells are present in human lymph nodes and are activated by $T$ cell-derived IL-2: a potential new link between adaptive and innate immunity. Blood. 101:3052-3057.

31. Freud, A.G., et al. 2005. A human CD34(+) subset resides in lymph nodes and differentiates into CD56bright natural killer cells. Immunity. 22:295-304.

32. Martin-Fontecha, A., et al. 2004. Induced recruitment of NK cells to lymph nodes provides IFN-gamma for $\mathrm{T}(\mathrm{H}) 1$ priming. Nat. Immunol. 5:1260-1265.

33. Paschen, A., et al. 2006. The coincidence of chromosome 15 aberrations and beta2-microglobulin gene mutations is causative for the total loss of human leukocyte antigen class I expression in melanoma. Clin. Cancer Res. 12:3297-3305.

34. Albert, D.M., et al. 1984. Establishment of cell lines of uveal melanoma. Methodology and characteris- tics. Invest. Ophthalmol. Vis. Sci. 25:1284-1299.

35. Kan-Mitchell, J., Mitchell, M.S., Rao, N., and Liggett, P.E. 1989. Characterization of uveal melanoma cell lines that grow as xenografts in rabbit eyes. Invest. Ophthalmol. Vis. Sci. 30:829-834.

36. Huang, X.Q., Mitchell, M.S., Liggett, P.E., Murphree, A.L., and Kan-Mitchell, J. 1994. Non-fastidious, melanoma-specific CD8+ cytotoxic T lymphocytes from choroidal melanoma patients. Cancer Immunol. Immunother. 38:399-405.

37. Repp, A.C., Mayhew, E.S., Apte, S., and Niederkorn, J.Y. 2000. Human uveal melanoma cells produce macrophage migration-inhibitory factor to prevent lysis by NK cells. J. Immunol. 165:710-715.

38. Ruggeri, L., et al. 2002. Effectiveness of donor natural killer cell alloreactivity in mismatched hematopoietic transplants. Science. 295:2097-2100.

39. Halfteck, G.G, et al. 2009. Enhanced in vivo growth of lymphoma tumors in the absence of the NKactivating receptor NKp46/NCF1. J. Immunol. 182:2221-2230

40. Gilfillan, S., et al. 2008. DNAM-1 promotes activation of cytotoxic lymphocytes by nonprofessional antigen-presenting cells and tumors. J. Exp. Med. 205:2965-2973.

41. Iguchi-Manaka, A., et al. 2008. Accelerated tumor growth in mice deficient in DNAM-1 receptor. J. Exp. Med. 205:2959-2964. 